\title{
TOPICS IN NONCOMMUTATIVE GEOMETRY INSPIRED PHYSICS
}

\author{
Rabin Banerjee ${ }^{1}$, Biswajit Chakraborty ${ }^{1}$, Subir Ghosh $^{2}$, Pradip Mukherjee ${ }^{3}$, Saurav Samanta $^{4}$ \\ ${ }^{1}$ S. N. Bose National Centre for Basic Sciences, JD Block, Sector III, Salt Lake, \\ Kolkata-700098, India \\ ${ }^{2}$ Physics and Applied Mathematics Unit, Indian Statistical Institute, Kolkata-700108, India \\ ${ }^{3}$ Presidency College, 86/1 College Street, Kolkata-700073, West-Bengal, India \\ ${ }^{4}$ Narasinha Dutt College, 129, Belilious Road, Howrah-711101, India
}

\begin{abstract}
In this review article we discuss some of the applications of noncommutative geometry in physics that are of recent interest, such as noncommutative many-body systems, noncommutative extension of Special Theory of Relativity kinematics, twisted gauge theories and noncommutative gravity.
\end{abstract}




\section{Table of Contents :}

1. Introduction 3

2. Noncommutative Theories, Symmetries and Their Implications 5

2.1 Elementary Idea About Moyal Star Product in 2D and the Landau Problem 5

2.2 Problem Regarding Poincare/Galilean symmetry and its restoration 6

2.3 Operatorial Approach $\quad 10$

3. Doubly Special Relativity and Noncommutativity 11

3.1 DSR Phase Space $\quad 12$

3.2 Canonical Variables $\quad 15$

3.3 Deformed Symmetry Generators 16

$\begin{array}{ll}3.4 \text { Lagrangian for } \kappa \text {-Particle } & 17\end{array}$

4. Deformed and Twisted Gauge Symmetry in Noncommutative Field Theory 18

4.1 Lagrangian Analysis $\quad 19$

4.2 Hamiltonian Analysis 26

5. Noncommutative Gravity and Black Hole Physics 30

5.1 Lie Algebraic Noncommutative Gravity 31

5.2 Noncommutativity Inspired Black Hole Physics 34

5.3 Coherent State Based Approach to Noncommutative Black Hole Physics 36

6 Concluding Remarks $\quad 42$

Acknowledgments $\quad 43$ 


\section{Introduction}

Preparing a review article on Non-Commutative (NC) geometry in physics turns out to be a daunting task mainly because of its great impact in diverse areas of modern physics such as Quantum Mechanics, High Energy, Gravity and Condensed Matter. There are excellent reviews [1] that cover much of the earlier work. In the present article we will concentrate on specific topics broadly falling in each of the above branches.

NC spacetime was first introduced by Snyder [2],

$$
\left[x_{\mu}, x_{\nu}\right]=i \theta_{\mu \nu}(x, p)=i \theta\left(x_{\mu} p_{\nu}-x_{\nu} p_{\mu}\right)
$$

in an attempt to introduce a short distance cutoff (the NC parameter) in a Lorentz covariant way that can cure the divergences in relativistic Quantum Field Theory (QFT). In (11) $x_{\mu}, p_{\mu}$ are the coordinate and momentum variables and $\theta$ is the $\mathrm{NC}$ parameter. However it did not become popular mainly due to its inability to address the radiative corrections correctly that were later accounted for successfully in the renormalization programme. Indeed, even at the very beginning Yang [3] had hinted about such a possibility. The remarkable rise in the interest in NC physics started with the seminal paper by Seiberg and Witten [4] where it was established that in certain low energy limits String Theory can be formulated as an effective QFT in NC spacetime,

$$
\left[x_{\mu}, x_{\nu}\right]=i \theta_{\mu \nu} .
$$

Still earlier, noncommutative spacetime was also derived from open string boundary conditions in [5]. In (2) $\theta_{\mu \nu}$ is generally taken as an antisymmetric constant tensor. A similar form of $\mathrm{NC}$ structure has been shown to arise from the consideration of boundary condition in the case of open (Super)String or membrane moving in the background of a constant antisymmetric tensor field, both at the classical [6] and quantum [7] level. Notice a qualitative difference between the forms of $\mathrm{NC}$ in (11) and (2): in the former $\mathrm{NC}$ is operatorial (it is basically the angular momentum operator) or dynamical whereas in (2) it of a $c$-number form. In the present article we refer to theories defined on $\mathrm{NC}$ spacetime as $\mathrm{NC}$ theories.

Subsequently many peculiar non-perturbative features of QFT in NC spacetime (2)), (these are essentially non-local effects), were revealed, such as NC solitons without a smooth commutative limit [8], formal similarity of abelian NC gauge theories with non-abelian gauge theories [1], (an example being the quantization of Chern-Simons coupling in abelian NC gauge theory [9]), structural similarity of NC gauge theories with gravity [10, 11] to name a few. On the other hand, following a prescription (the Seiberg-Witten map) given in [4] on how to obtain $\mathrm{NC}$ corrections in perturbative QFT framework order by order in the NC parameter $\theta$ a large amount of work has appeared that studied NC extensions of QFT anomalies [12], NC-solitons having smooth commutative limit [13], Chern-Simons gauge theories [14], (for more recent result valid to all orders of $\theta$ see [15] that follow the general method proposed by [16]) etc. NC generalization in Quantum Mechanical models such as Harmonic Oscillator [17, Hydrogen atom spectra [18], NC gravitational well [19] are geared to predict theoretical values of $\theta$ that can

be checked experimentally. However, perturbative analysis also generates a problem, known as the Ultra violet - Infrared mixing [20], that shows that high and low energy scales of energy get 
entangled due to noncommutativity which can affect the renormalization programme. So far there is no conclusive experimental evidence for a non-vanishing $\theta$. To name some of important and active areas (that we have not touched upon in this review) we mention renormalization for NCQFT [21], NC geometry in the context of spectral model of spacetime [22], NC description of Quantum Hall Fluid [23], among others.

More recently, the operatorial form of NC spacetime (11) or a Lie algebraic form, (where $\theta_{\mu \nu}(x)$ depends only on $\left.x_{\mu}\right)$, is playing important roles in different contexts. An example is in Condensed Matter Physics where planar systems involving a perpendicular magnetic field becomes effectively non-commutative in the lowest Landau level [24] (see Szabo in [1]) the NC parameter being identified with inverse of the magnetic field or in Anyon models [25]. The Landau levels also get renormalized by interparticle interactions [26] that can have nontrivial impact in fractional Quantum Hall Effect. In another development it has been shown that NC phase space algebra can influence particle dynamics directly via induced Berry curvature effects [27] in studying many Condensed Matter phenomena such as Anomalous Hall effect [28, 24], Spin Hall effect [29], models of Graphene [30] among others. NC momentum algebra was used in [31] to rederive the Dirac Quantization Condition in a gauge invariant way and this result has been extended in $\mathrm{NC}$ space in a recent work [32].

Indeed, our review is biased in the sense that we have left out a number of exciting areas in NC physics some of which are mentioned above. Our listing is, by no means, exhaustive. This review is restrictive and presents our perspective and choice of topics. However, in this brief Introduction, we will not dwell too much on the topics we have covered in the present review simply because the individual chapters are reasonably self-contained. Section 2 introduces the star (Moyal) product used to define products of fields in NC spaces discusses effects of NC space on non-relativistic many (bose/fermi) particle systems through twisted statistics [26, 33, 34, 35]. In Section 3 we further develop kinematics in NC spacetime at an algebraic level [36] by considering the Doubly Special Relativity (DSR) framework from the perspective of NC spacetime. The concept of DSR, proposed by Amelino Camelia [37] (as an extension to Special Theory of Relativity), can act as the proper arena for Quantum Gravity, since it can accommodate an observer independent length scale (which might be Planck length). Section 4 gives a detailed analysis of gauge symmetries in NC gauge theories, pointing out the similarities and differences with the conventional interpretation in ordinary (commutative space) gauge theories [38, 39]. The idea goes back to the approach proposed by Wess [40] and by Chaichian et.al [41, 42] who showed that one can interpret the Lorentz (or Poincare) symmetry violating NC theories to be invariant under a Twisted Lorentz (or Poincare) symmetry 1 . Section 5 is devoted to a study of NC effects in Gravity [44, 45] where Seiberg-Witten map is utilized in computing $O(\theta)$ effects on Einstein Gravity. For an alternative approach based on coherent state formalism [46] see [47]. We end with concluding remarks in Section 6.

\footnotetext{
${ }^{1}$ For a discussion on Poincare symmetry in NC field theory using Noether prescription see 43.
} 


\section{Noncommutative Theories, Symmetries and Their Im- plications}

In this section, we are going to review very briefly the Moyal type NC space in a nonrelativistic setting touching on important applications in Quantum Hall Effect (QHE), violation and eventual restoration of rotational symmetry in space with dimension $D \geq 3$. This serves the dual purpose of how dramatically this impacts on the deformation of Bose/Fermi statistics giving rise to the violation of Pauli's principle in one hand and on the other hand it illustrates how the Poincare symmetry is broken and eventually restored in straightforward manner. This restoration of Poincare symmetry is an essential pre-requisite for the elementary particles to be classified according to Wigner.

\subsection{Elementary Idea About Moyal Star Product in 2D and the Lan- dau Problem}

To motivate briefly the introduction of Moyal star product formalism let us consider the two dimensional noncommutative plane

$$
\begin{gathered}
{\left[\hat{x}_{1}, \hat{x}_{2}\right]=i \theta} \\
{\left[\hat{t}, \hat{x}_{i}\right]=0 ; i=1,2}
\end{gathered}
$$

so that time 't' can be regarded as a usual commutating parameter. At this stage, one can go ahead with the quantization programme by working with the operator-valued coordinates (see sub-section 5.3) or else demote the status of the operators ' $\hat{x}_{1}$ ' and ' $\hat{x}_{2}$ ' to ordinary c-numbered valued coordinates, where the compositions of any pair of functions thereof has to be performed by the Moyal star product [1]. To see this, heuristically consider the Weyl's prescription of constructing Weyl ordered operator $W[f(\vec{x})]$ from the c-numbered valued function $f(\vec{x})$ of the commuting variables $x_{1}, x_{2}$. According to this prescription, one has to just replace $\vec{x}$ occurring in the exponent in the identity

$$
f(\vec{x})=\frac{1}{(2 \pi)^{2}} \int d^{2} k d^{2} y e^{i \vec{k} \cdot(\vec{x}-\vec{y})} f(\vec{y})
$$

by the corresponding operator $\hat{\vec{x}}$ :

$$
W\left[f\left(x_{i}\right)\right]=\frac{1}{(2 \pi)^{2}} \int d^{2} k d^{2} y e^{i \vec{k} \cdot(\hat{\vec{x}}-\vec{y})} f(\vec{y}) .
$$

For example, if $f(\vec{x})=x_{1} x_{2}$, then one can easily show that

$$
W\left[x_{1} x_{2}\right]=\frac{1}{2}\left(\hat{x}_{1} \hat{x}_{2}+\hat{x}_{2} \hat{x}_{1}\right) .
$$


One can then show that for any pair of such functions $f\left(x_{i}\right)$ and $g\left(x_{i}\right)$ the composition rule between them should be modified appropriately to the Moyal star product $\left(^{*}\right)$, so that

$$
W\left[f\left(x_{i}\right)\right] W\left[g\left(x_{i}\right)\right]=W\left[(f * g)\left(x_{i}\right)\right]
$$

where

$$
(f * g)\left(x_{i}\right)=\left.e^{\frac{i}{2} \theta \epsilon_{i j} \partial_{i}^{x} \partial_{j}^{y}} f\left(x_{i}\right) g\left(y_{i}\right)\right|_{y=x} .
$$

For a more general formulation see [48. It is quite easy to verify the Moyal brackets defined through this star product, between the c-numbered valued coordinates now becomes isomorphic to the commutators of their corresponding operators (3)

$$
\left[x_{1}, x_{2}\right]_{*}=x_{1} * x_{2}-x_{2} * x_{1}=i \theta \text {. }
$$

Although not commutative, the Moyal star product is associative.

This 2D noncommutative problem arises naturally in the Landau problem, where a charged particle (say an electron) moving in $x y$ plane and subjected to a transverse magnetic field 'B' along the z-direction. In this problem, which plays a central role in QHE the commuting $\hat{x}_{1}$ and $\hat{x}_{2}$ coordinates fails to commute, when projected to the lowest Landau level [1, 49]

$$
\left[P \hat{x}_{1} P, P \hat{x}_{2} P\right]=\frac{1}{i B}
$$

so that the noncommutativity is given by $\left(\frac{1}{B}\right)$. This noncommutativity implies that the guiding center coordinates in the Lowest Landau Level satisfy the usual uncertainty relations

$$
\Delta x_{1} \Delta x_{2} \geq \frac{1}{B}
$$

This uncertainty relations between the projected coordinates (here we have intentionally suppressed the projection operator $P$ ) indicates that the minimal area occupied by any particle is $\approx \frac{1}{B}$ in Lowest Landau Level and, thus for fermions there exists an upper bound to the number of particles that can be accommodated in unit area. On the other hand, interparticle interactions can renormalise the noncommutative parameter away from $\left(\frac{1}{B}\right)$ and thus can have affect on the filling fraction in a Quantum Hall system. Indeed, it has been demonstrated that Jain fraction for Fractional Quantum Hall Effect can be obtained in a heuristic treatment, where the electrons are attached to appropriate magnetic flux tubes [26]. Furthermore, there are certain situations where interactions can be traded with noncommutativity within a certain approximation, as can be seen by constructing a dual families of noncommutative quantum systems [50].

\subsection{Problem Regarding Poincare/Galilean Symmetry and its Restora- tion}

The expression of Moyal star-product given above (7) can be generalised to $3+1$ dimensional spacetime as

$$
(f * g)(x)=\left.e^{\frac{i}{2} \theta^{\mu \nu} \partial_{\mu}^{x} \partial_{\nu}^{y}} f(x) g(y)\right|_{y=x}
$$


However, the introduction of the length scale through the NC matrix $\Theta=\left\{\theta^{\mu \nu}\right\}$ in more than two-dimension violates Poincare or more precisely, the symmetry under homogeneous Lorentz transformation. This can be understood easily by considering the transformation property of scalar field $\phi(x)$ under a homogeneous Lorentz transformation

$$
\begin{aligned}
x^{\mu} & \rightarrow x^{\prime \mu}=\Lambda_{\nu}^{\mu} x^{\nu} \\
\phi & \rightarrow \phi^{\Lambda}(x)=\phi\left(\Lambda^{-1} x\right)
\end{aligned}
$$

One can then easily see that for a pair of arbitrary scalar fields $\phi_{1}(x)$ and $\phi_{2}(x)$, the automorphism under the homogeneous Lorentz group does not hold

$$
\left(\phi_{1}^{\Lambda} * \phi_{2}^{\Lambda}\right)(x) \neq\left(\phi_{1} * \phi_{2}\right)^{\Lambda}(x)
$$

Infact the easiest way to understand (14) is through (2). (Note that the translational symmetry is not affected and like-wise in $D=2$ case, considered above, the $S O(2)$ symmetry is also not violated, as $\theta_{i j}$ behaves as an $S O(2)$ scalar.). This symmetry can, however, be restored by using an appropriate Drinfeld twist in a Hopf algebraic framework. To illustrate the essential ideas involved, let us consider the case of $S O(3)$ symmetry itself in $R^{3}$, where we take $\theta^{0 i}=0$. Being a subgroup also of the Galilean group, this demonstrates how the Galilean symmetry can also be restored in the framework. In fact, even in the presence of a nonvanishing $\theta^{0 i}$ the broken symmetry under Galilean boost is taken care of rather trivially [33]. To begin with, one interprets the $\mathrm{NC} *$-product introduced in (11) as to correspond $\mathrm{NC}$ algebra $\mathcal{A}_{\theta}$, obtained by deforming the commutative algebra $\mathcal{A}_{o}$, where fields compose through point-wise multiplication, i.e effectively with $\theta=0$.

More formally, the multiplication map ' $m_{\theta}$ ' in $\mathcal{A}_{\theta}$ is obtained from ' $m_{o}$ ' in $\mathcal{A}_{o}$

$$
\begin{aligned}
m_{o}: \mathcal{A}_{o} \otimes \mathcal{A}_{o} & \rightarrow \mathcal{A}_{o} \\
m_{o}(f(x) \otimes g(x)) & =f(x) g(x)
\end{aligned}
$$

by inserting a twist operator $\mathcal{F}_{\theta} \equiv e^{-\frac{i}{2} \theta^{i j} P_{i} \otimes P_{j}} \in \mathcal{U}(I S O(3)) \otimes \mathcal{U}(I S O(3))$ as

$$
\begin{aligned}
m_{\theta}: \mathcal{A}_{\theta} \otimes \mathcal{A}_{\theta} & \rightarrow \mathcal{A}_{\theta} \\
m_{\theta}(f(x) \otimes g(x)) & =m_{0}\left(\mathcal{F}_{\theta}(f(x) \otimes g(x))\right)
\end{aligned}
$$

The operators $P_{i}$ used above are the generators of the translational algebra $T_{3}$. Here $I S O(3)=$ $S O(3) \ltimes T_{3}$ is a semi-direct product of $S O(3)$ and the translational algebra $T_{3}$ in $\mathbb{R}^{3}$ and $\mathcal{U}(I S O(3))$ is the corresponding universal enveloping algebra, which is a Hopf algebra. This suggest that one has to go beyond the usual Lie-algebraic framework to a (deformed) Hopf algebraic one to capture the relevant symmetries. Indeed, it can be easily shown that the usual co-product of the deformed Hopf algebra should also be deformed as [51, 52] (for a review see [34]).

$$
\Delta_{o}(\vec{J})=\vec{J} \otimes \mathbb{I}+\mathbb{I} \otimes \vec{J} \rightarrow \Delta_{\theta}(\vec{J})=\mathcal{F}_{\theta}^{-1} \Delta_{o}(\vec{J}) \mathcal{F}_{\theta}
$$

in order to be compatible with the deformed product ' $m_{\theta}$ ' (111):

$$
m_{\theta}\left[\Delta_{\theta}(\vec{J})(f \otimes g)\right]=\vec{J} \triangleright m_{\theta}(f \otimes g) .
$$


Here,$\vec{J}$ ' is taken to be the $S O(3)$ generators. Note that the co-product of the translational generators $\vec{P} \in T_{3}$ does not undergo any deformation by this abelian twist:

$$
\Delta_{o}(\vec{P}) \rightarrow \Delta_{\theta}(\vec{P})=\mathcal{F}_{\theta}^{-1} \Delta_{o}(\vec{P}) \mathcal{F}_{\theta}=\Delta_{o}(\vec{P})
$$

Besides, the other maps the antipode and the co-unit undergoes no deformation. An immediate consequence of this deformation is that two particle exchange map $\tau_{o}(\phi \otimes \psi)=\psi \otimes \phi$ and hence the corresponding projection operator $P_{o}=\frac{1}{2}\left(\mathbb{I} \pm \tau_{o}\right)$ into the symmetric/antisymmetric subspaces, describing bosons/fermions, also get deformed in a similar manner [35]

$$
\begin{aligned}
\tau_{o} & \rightarrow \tau_{\theta}=\mathcal{F}_{\theta}^{-1} \tau_{o} \mathcal{F}_{\theta} \\
P_{o} & \rightarrow P_{\theta}=\mathcal{F}_{\theta}^{-1} P_{o} \mathcal{F}_{\theta}
\end{aligned}
$$

In the process one introduces the, so-called, twisted boson/fermions through the deformed exchange operator $\tau_{\theta}$, ensuring super-selection principle, as

$$
\left[\Delta_{\theta}, \tau_{\theta}\right]=\left[\Delta_{\theta}, P_{\theta}\right]=0
$$

Furthermore, it has also been shown that $\theta_{i j}$ indeed transforms as a scalar under the action of $\mathrm{SO}(3)$, when it is implemented through the above twisted co-product [51]

$$
\vec{J}_{\theta} \triangleright \theta^{i j}=0
$$

where the subscript ' $\theta$ ' indicate that one has to consider the action of $\vec{J}$, through a twisted coproduct. Finally, let us demonstrate through a heuristic argument, how the algebra involving the corresponding creation/annihilation operators also get deformed [33, 53] which was observed for the first time in [35] .

To begin with, let us apply the twisted projection operator $P_{\theta}$ on the tensor product of two momentum eigenstates $(|k\rangle \otimes|l\rangle)$ to write it in the usual symmetric/antisymmetric form up to a phase factor as

$$
\left.\left.P_{\theta}(|k\rangle \otimes|l\rangle)=\frac{1}{2}\left(|k\rangle \otimes|l\rangle \pm \mathcal{F}_{\theta}^{-2}|l\rangle \otimes|k\rangle\right)=\frac{1}{2} e^{i k \wedge l}(|k, l\rangle\rangle \pm|l, k\rangle\right\rangle\right)
$$

where

$$
|k, l\rangle\rangle \equiv e^{-i k \wedge l}|k\rangle \otimes|l\rangle
$$

and

$$
k \wedge l=\frac{1}{2} \theta^{i j} k_{i} l_{j}
$$

Now identifying $a_{k}^{\dagger} a_{l}^{\dagger}|0\rangle=P_{\theta}(|k\rangle \otimes|l\rangle)$, it easily follows that

$$
a_{k}^{\dagger} a_{l}^{\dagger}= \pm e^{2 i k \wedge l} a_{l}^{\dagger} a_{k}^{\dagger}
$$

The phase of the other commutation relation

$$
a_{k}^{\dagger} a_{l}= \pm\left(a_{l} a_{k}^{\dagger}-(2 \pi)^{3} \delta^{3}(\vec{k}-\vec{l})\right) e^{-2 i k \wedge l}
$$


can be understood easily from the fact that the annihilation operator $a_{l}$ is associated with momentum $(-l)$, in contrast to the operator $a_{l}^{\dagger}$, for which the associated momentum is $+l$. These operators, which satisfy a (anti)commutation relation turns out to be related to the undeformed $\left(\theta=0\right.$ ones through a $U(1)$ transformation as $a_{k}=a_{k}(\theta=0) \exp \left(\frac{i}{2} k \wedge P\right)$ where $P$ is the total momentum. For an interpretation through deformed Heisenberg algebra see [54].

These twisted (anti) commutation relations can have some of the drastic consequences. For example, it can violate Pauli's exclusion principle [33]. To illustrate this briefly, consider the density matrix of a canonical ensemble, comprising a pair of free identical twisted fermions/bosons:

$$
\rho=e^{-\beta H} ; H=\frac{1}{2 m}\left(\vec{P}^{2} \otimes \mathbb{I}+\mathbb{I} \otimes \vec{P}^{2}\right) .
$$

The 2-particle correlation function in the "thermodynamical" limit is then obtained as

$$
C\left(r=\left|\overrightarrow{r_{1}}-\overrightarrow{r_{2}}\right|\right)=\frac{1}{Z}\left(\left(\left\langle\overrightarrow{r_{1}}\right| \otimes\left\langle\overrightarrow{r_{2}}\right|\right) P_{\theta} \rho P_{\theta}\right)\left(\left|\overrightarrow{r_{1}}\right\rangle \otimes\left|\overrightarrow{r_{2}}\right\rangle\right)
$$

which represents the probability density of a particle to be detected around $\overrightarrow{r_{1}}$, given that the other is at $\overrightarrow{r_{2}}$. This in turn relates to the effective (statistical) potential $V_{\text {eff }}(r)$ as

$$
C(r)=e^{-\beta V_{e f f}(r)}
$$

(see [33] and references there in).

On explicit computation, one easily finds that for a $2 D$ system a large area $A$, this is given by

$$
C(r)=\frac{1}{A^{2}}\left[1 \pm \frac{1}{1+\frac{\theta^{2}}{\lambda^{4}}} e^{\frac{-2 \pi r^{2}}{\left[\lambda^{2}\left(1+\frac{\theta^{2}}{\lambda^{4}}\right)\right]}}\right]
$$

where $\lambda=\sqrt{\frac{2 \pi \beta}{m}}$ is the thermal wavelength and $\beta=\frac{1}{k T}$ and \pm sign referes to twisted bosonic and fermionic cases respectively.

The profile of $V_{\text {eff }}(r)$ for twisted fermions (TF) and the conventional fermions $(\theta=0)$ shows that the $V_{\text {eff }}$ saturates to a finite value (soft core potential) for coincident points of a part of twisted fermions, unlike the usual case, where it diverges (hard core). This demonstrates that two fermions can indeed sit on the top of each other, in principle, violating Pauli's exclusion principle, although it requires an enormous energy to do so. For other effects, see [34].

We would, however, like to mention here that there were some controversies recently in the literature on this issue of twisted statistics. Particularly in [55] it was pointed out that one should implement braided twisted symmetry, rather than the usual twisted symmetry. In this scheme one is entitled to define $*$-product between fields even at distinct spacetime points, which virtually washes out any noncommutative effect. In particular, the twisted symmetric fermions or bosons do not occur here. In fact, it has been shown in [53] that the energy shift of noncommutative origin does not appear in the system of degenerate electron gas, if braided twisted symmetry is implemented, whereas it occurs in the case of usual twisted symmetry. But this was again criticised in a recent work [56] (see also [57]). 


\subsection{Operatorial Approach}

So far, we have been dealing with Moyal star product formalism in dealing with the various quantum mechanical/field theoretical systems. On the other hand, recently a formalism is being developed [58] in the context of noncommutative quantum mechanics, where one confronts the operatorial nature of the coordinate variables "head on," rather than 'demoting' them to ordinary c-number variables and use Moyal star product 2 . So far this has been sorted out for the spatial dimension $D=2$ only and work is in progress to extend the formalism to $D=3$ and beyond. To cite examples two major accomplishments of the framework is that it has been possible to find the exact energy eigenvalues of a simple quantum mechanical problem of a particle confined in an infinite spherical potential well. It will indeed be very difficult, if not impossible, to obtain the same in a Moyal product formalism. Besided in [59] the authors have provided a path-integral representation of the transition amplitude, using coherent state approach, and obtained the form of a non-local action of a particle moving in noncommutative plane subjected to arbitrary potential.

In the following we outline briefly the essential ideas involved. To begin with, one observes that the structure of the coordinate algebra $[\hat{x}, \hat{y}]=i \theta$ in $D=2$ case, is isomorphic to the phase space Heisenberg algebra $[\hat{x}, \hat{p}]=i \hbar$ of $1-D$ harmonic oscillator so that here $\hat{y}$ plays the role of $\hat{p}$, while $\theta$ plays the role of $\hbar$. Consequently the classical configuration space $\mathcal{H}_{c}$ in the $\mathrm{NC}$ case is just isomorphic to the bosonic Fock space of the harmonic oscillator. One thus defines the classical configuration space as

$$
\mathcal{H}_{c}=\operatorname{Span}\{|n\rangle\}_{n=0}^{\infty}
$$

where the span is taken over the field of complex numbers and

$$
|n\rangle=\frac{1}{\sqrt{n !}}\left(b^{\dagger}\right)^{n}|0\rangle ; \quad b=\frac{1}{\sqrt{2 \theta}}(\hat{x}+i \hat{y})
$$

and $|0\rangle$ satisfies $b|0\rangle=0$ by definition.

The quantum Hilbert space, (the set of all quantum states) is identified with

$$
\mathcal{H}_{q}=\left\{\psi(\hat{x}, \hat{y}): \operatorname{tr}_{c}\left(\psi^{\dagger}(\hat{x}, \hat{y}) \psi(\hat{x}, \hat{y})\right)<\infty\right\}
$$

In other words, the Hilbert space is the trace class enveloping algebra of the classical configuration space $\mathcal{H}_{c}$ Fock algebra $\left(b, b^{\dagger}\right)$. As these operators are necessarily bounded, this is again a Hilbert space (recall that the set of all bound operators in a Hilbert space is again a Hilbert

\footnotetext{
${ }^{2}$ It should be recalled that there is an essential difference between quantum mechanics and quantum field theory, as far as the status of the position coordinates are concerned: Even in the ordinary commutative $(\theta=0)$ theories, the role of position coordinates $\hat{x}_{i}$ is that of an hermitian observable, whereas in quantum field theory these are mere labels of the continuous degrees of freedom and therefore they do not belong to the configuration space and do not satisfy any Heisenberg algebra. Interestingly, this difference carries over (as expected) to the noncommutative counterparts. Although the status of $x_{i}$ 's is upgraded to an operator $\hat{x}_{i}$ in noncommutative theories it is definitely not valued in the same vector space as that of the field operators in noncommutative quantum field theories.
} 
space). We denote the states in $\mathcal{H}_{c}$ by $|\cdot\rangle$, whereas a state in $\mathcal{H}_{q}$ will be denoted by |.). The corresponding inner product is $(\psi \mid \phi)=(\psi, \phi)=\operatorname{tr}_{c}\left(\psi^{\dagger} \phi\right)$, which also serves to define bra states as elements of the dual space (linear functionals). Note that the trace is performed over the classical configuration space, denoted by the subscript ' $c$ '.

The next step is to construct a representation [60, 61] of the NC Heisenberg algebra

$$
\left[\hat{x}_{i}^{q}, \hat{x}_{j}^{q}\right]=i \theta \epsilon_{i j} ; \quad\left[\hat{x}_{i}, \hat{p}_{j}^{q}\right]=i \hbar \delta_{i j}, ; \quad\left[\hat{p}_{i}^{q}, \hat{p}_{j}^{q}\right]=0 \quad(i, j=1,2)
$$

on $\mathcal{H}_{q}$. This is done simply by defining the action of these operators as follows:

$$
\begin{array}{r}
\hat{x}_{i}^{q} \psi(\hat{x}, \hat{y})=\hat{x}_{i} \psi(\hat{x}, \hat{y}) \\
\hat{p}_{i}^{q} \psi(\hat{x}, \hat{y})=\frac{\hbar}{\theta} \epsilon_{i j}\left[\hat{x}_{j}, \psi(\hat{x}, \hat{y})\right]
\end{array}
$$

where $\psi(\hat{x}, \hat{y})$ is an arbitrary operator in the $\mathcal{H}_{q}$. Note that the momenta act as inner automorphisms with respect to $\hat{x}_{i}$ and $\hat{x}_{i}$ 's are taken to act through left multiplication. It can now be trivially verified, by using the NC Heisenberg algebra (36), the definition of the inner product $(\psi \mid \phi)$ and the Jacobi identity, that it, in fact, furnishes a unitary representation. For quantum mechanical interpretation of this formalism, see 62].

Using this formalism, the complete spectrum of a particle, confined in a $\mathrm{NC}$ spherical well in $D=2$ could be obtained [58]. The bound and scattering states for finite potential barrier could also be obtained unambiguously. It was also shown that the time-reversal symmetry is broken by noncommutativity, which can only be restored in the commutative or thermodynamic limit. Based on this, the thermodynamics of an ideal Fermi gas in this infinite spherical well has also been studied [63], which exhibits some of the remarkable behaviors implied by the excluded area resulting from the noncommutativity. In particular, there are extremal macroscopic states, characterized by area, number of particles and angular momentum, that correspond to a single microscopic state and thus have vanishing entropy. Furthermore, for comparable system size and excluded area, the thermodynamical quantities, such as entropy, exhibit non-extensive features.

\section{Doubly Special Relativity and Noncommutativity}

The idea of Doubly (or Deformed) Special Relativity (DSR), formulated by Amelino-Camelia [37] was a culmination of several apparently disconnected issues. The combined wisdom of theorists demanded a radical departure from conventional physics in the regime of Planck scale [64. It appeared [65] that one needs to modify the (Einsteinean ) energy momentum dispersion law, a possible form being $E^{2}=c^{2} \vec{p}^{2}+c^{4} m^{2}+\eta L_{P}^{n} c^{2} \vec{p}^{2} E^{n}+\ldots$, where $\eta$ is a numerical factor and $L_{P}$ is a fundamental length scale, which can be Planck length. The modification has to be such that at low energy the standard relation $E^{2}=c^{2} \vec{p}^{2}+c^{4} m^{2}$, compatible with Special Theory (SR), is recovered.

But this leads to the first clash with SR because (Planck) length or energy are not observer independent quantities. In SR $c$ is the only observer independent scale. However we do not 
want to discard the Relativity Principle: The laws of physics take the same form in all inertial frames. The only way to achieve both the above is to generalize the SR (coordinate and momentum) transformation rules in such a way that two observer independent scales $c$ and $L_{P}$ (instead of the single one $c$ in SR) can be accommodated - hence Doubly (or Deformed) Special Relativity (DSR) [37]. The situation is similar to the transition from no scale and linear Galilean Relativity to one scale (c) SR where the velocity addition theorem becomes non-linear. In a similar vein the transition from SR to DSR yields two scales ( $c$ and $L_{P}$ ) at the cost of ushering another level of non-linearity, that in the energy momentum transformation rules. In fact we will see that the phase space variable transformation rules and invariants under DSR laws get completely entangled [66, 36].

However there is another very important feature of DSR: it is intimately connected to a Non-Commutative spacetime, the $\kappa$-Minkowski spacetime [37, 67]. This is indeed nice because a theory claimed to be valid at Planck length has to have an inherent noncommutativity because the Quantum Gravity models 64] predict a foam like discrete spacetime below Planck length. Furthermore the coexistence of black hole physics and quantum mechanics also demands a discrete spacetime [68].

Since our focus is on the NC aspect of DSR we will not discuss the DSR itself anymore (for which there are reviews [69]). We will concentrate more on the NC aspect of DSR. Again there are two popular ways to introduce noncommutativity in DSR scenario: (I) The $\kappa$-Poincare Hopf algebra approach [70] and the $\kappa$-Minkowski spacetime approach [67]. In the former the Poincare Lie algebra framework of SR is extended to a Hopf algebra. In the latter one tries to keep the SR Poincare algebra intact by changing the Poincare generators and transformation laws appropriately. One advantage of the latter [36] is that the Lorentz group theoretic classifications of the quantum fields in DSR will possibly remain unchanged. In the present article we will follow the second alternative.

\subsection{DSR Phase Space}

In the sense of classical Poisson Brackets, the NC $\kappa$-Minkowski spacetime is defined as [37, 67, 36.

$$
\left\{x^{i}, x^{0}\right\}=\frac{x^{i}}{\kappa} ; \quad\left\{x^{i}, x^{j}\right\}=0 .
$$

The form of of modified dispersion relation, we will consider was proposed by Magueijo and Smolin [66] (MS)

$$
p^{2}=m^{2}\left[1-\frac{E}{\kappa}\right]^{2}=m^{2}\left[1-\frac{(\eta p)}{\kappa}\right]^{2}
$$

with $E$ being the particle energy and $(\eta p)=\eta^{\mu} p_{\mu}, \eta_{0}=1, \eta_{i}=0$. Here the metric is diagonal with components $g^{00}=-g^{i i}=1$.

It should be mentioned that, even if one imposes the restrictions that Jacobi identities have to be maintained and that the structure should reduce to canonical algebra for $\kappa \rightarrow \infty$, the full $\kappa$-NC phase space algebra is not uniquely determined. There are distinct (and possibly inequivalent) representations that are connected by non-linear transformations [71]. This is 
possible because DSR formalism can be interpreted as a nonlinear realization of the Lorentz group. It is interesting to point out that for a particular combination of variables [71 the DSR algebra becomes isomorphic to the Snyder algebra [2]. However the sector of the algebra common to all forms of $\kappa$-Minkowski spacetime is

$$
\left\{x^{i}, x^{0}\right\}=\frac{x^{i}}{\kappa} ;\left\{x^{i}, x^{j}\right\}=0 ; \quad\left\{x^{i}, p^{j}\right\}=-g^{i j} ;\left\{p^{\mu}, p^{\nu}\right\}=0 .
$$

The particular $\kappa$-NC phase space that we will use here was first studied and further developed in [72] (in a restricted set up of 1+1-dimensional toy model). In fact this phase space can be extracted from very general deformations considered by Lukierski et.al. [67]. Rest of the phase space algebra is given below,

$$
\left\{x^{0}, p^{i}\right\}=p^{i} / \kappa ;\left\{x^{i}, p^{0}\right\}=0 ;\left\{x^{0}, p^{0}\right\}=-1+p^{0} / \kappa .
$$

The above is rewritten in a covariant form,

$$
\begin{gathered}
\left\{x_{\mu}, x_{\nu}\right\}=\frac{1}{\kappa}\left(x_{\mu} \eta_{\nu}-x_{\nu} \eta_{\mu}\right), \\
\left\{x_{\mu}, p_{\nu}\right\}=-g_{\mu \nu}+\frac{1}{\kappa} \eta_{\mu} p_{\nu}, \quad\left\{p_{\mu}, p_{\nu}\right\}=0 .
\end{gathered}
$$

We wish to construct the finite Lorentz transformation (LT) consistent with this NC space. These were first constructed in [73]. We will instead follow another more systematic route that was exploited in [74, 36]. We need the rotation generators to generate infinitesimal variations. The angular momentum is defined in the normal way as,

$$
J_{\mu \nu}=x_{\mu} p_{\nu}-x_{\nu} p_{\mu} .
$$

This is motivated by the fact that spatial sector of $\kappa$-NC algebra in (42) remains unaffected. Furthermore, using (42) one can check that the Lorentz algebra is intact,

$$
\left\{J^{\mu \nu}, J^{\alpha \beta}\right\}=g^{\mu \beta} J^{\nu \alpha}+g^{\mu \alpha} J^{\beta \nu}+g^{\nu \beta} J^{\alpha \mu}+g^{\nu \alpha} J^{\mu \beta} .
$$

However, Lorentz transformations of $x_{\mu}$ and $p_{\mu}$ are indeed affected,

$$
\left\{J^{\mu \nu}, x^{\rho}\right\}=g^{\nu \rho} x^{\mu}-g^{\mu \rho} x^{\nu}+\frac{1}{\kappa}\left(p^{\mu} \eta^{\nu}-p^{\nu} \eta^{\mu}\right) x^{\rho} ;\left\{J^{\mu \nu}, p^{\rho}\right\}=g^{\nu \rho} p^{\mu}-g^{\mu \rho} p^{\nu}-\frac{1}{\kappa}\left(p^{\mu} \eta^{\nu}-p^{\nu} \eta^{\mu}\right) p^{\rho} .
$$

Notice that the extra terms appear for $J^{0 i}$ and not for $J^{i j}$ so that only boost transformations are changed.

From now on we will use the $(x, y, z, t)$ notation (instead of the covariant one), which is more suitable for comparison with existing results. We define the infinitesimal transformation of a generic variable $O$ by,

$$
\delta O=\left\{\frac{1}{2} \omega_{\mu \nu} J^{\mu \nu}, O\right\}
$$


and only the parameter $\omega_{0 x}=\delta u$ is non-vanishing.

Let us start with the energy-momentum vector $\left(E, p_{x}, p_{y}, p_{z}\right)$. The above considerations yield the following differential equations [36],

$$
\frac{d E}{d u}=-p_{x}+\frac{E p_{x}}{\kappa} ; \quad \frac{d p_{x}}{d u}=-E+\frac{p_{x}^{2}}{\kappa} ; \quad \frac{d p_{y}}{d u}=\frac{p_{y} p_{x}}{\kappa} ; \quad \frac{d p_{z}}{d u}=\frac{p_{z} p_{x}}{\kappa} .
$$

The details can be obtained from [36]. The final result is the $\kappa$-LT rules,

$$
E^{\prime}=\frac{\gamma\left(E-v p_{x}\right)}{\alpha} ; p_{x}^{\prime}=\frac{\gamma\left(p_{x}-v E\right)}{\alpha} ; p_{y}^{\prime}=\frac{p_{y}}{\alpha} ; p_{z}^{\prime}=\frac{p_{z}}{\alpha}
$$

where $\gamma=\frac{1}{\sqrt{1-v^{2}}}, \alpha=1+\frac{1}{\kappa}\left\{\left((\gamma-1) E-v \gamma p_{x}\right\}\right)$. Notice that, unlike SR LTs, in $\kappa$-LT the components transverse to the velocity $v$ are also affected and the $\kappa$-effect appears as the factor $\alpha$ and for $\kappa=\infty, \alpha=1$ so that SR LTs are recovered.

Before proceeding to derive the $\kappa$-LT for the coordinates $x_{\mu}$, let us first find out the new dispersion law that is $\kappa$-LT invariant. Scanning the following infinitesimal transformation rules,

$$
\left\{\frac{1}{2} J_{\mu \nu}, p^{2}\right\}=\frac{p^{2}}{\kappa}\left(\eta_{\mu} p_{\nu}-\eta_{\nu} p_{\mu}\right) ; \quad\left\{\frac{1}{2} J_{\mu \nu},(\eta p)\right\}=-\left(1-\frac{(\eta p)}{\kappa}\right)\left(\eta_{\mu} p_{\nu}-\eta_{\nu} p_{\mu}\right) ;
$$

we find the following combination to be invariant:

$$
\left\{\frac{1}{2} J_{\mu \nu}, \frac{p^{2}}{\left(1-\frac{(\eta p)}{\kappa}\right)^{2}}\right\}=0 .
$$

The finite $\kappa$-LTs also yields

$$
\left(p^{2}-m^{2}\left(1-\frac{(\eta p)}{\kappa}\right)^{2}\right)^{\prime}=\frac{1}{\alpha^{2}}\left(p^{2}-m^{2}\left(1-\frac{(\eta p)}{\kappa}\right)^{2}\right),
$$

confirming that the new $\kappa$-LT invariant dispersion law is

$$
p^{2}=m^{2}\left(1-\frac{(\eta p)}{\kappa}\right)^{2}
$$

This is the MS dispersion law [66] (39).

In an identical fashion, putting the coordinates for $O$ in (3) we compute the $\kappa$-LTs for the coordinates [73, 36],

$$
t^{\prime}=\alpha \gamma(t-v x) ; \quad x^{\prime}=\alpha \gamma(x-v t), \quad y^{\prime}=\alpha y, \quad z^{\prime}=\alpha z .
$$

Once again we notice the similar features as in the momentum transformation laws.

As in the dispersion relation, we look for an invariant quantity that will generalize the conventional distance and we find that under the $\kappa$-LT (53),

$$
\left(x^{2}\left(1-\frac{(\eta p)}{\kappa}\right)^{2}\right)^{\prime}=x^{2}\left(1-\frac{(\eta p)}{\kappa}\right)^{2} .
$$


Hence the invariant length is generalized to

$$
s^{2}=x^{2}\left(1-\frac{(\eta p)}{\kappa}\right)^{2} .
$$

This is one of the important results of [36] that can have connections with Finsler geometry [75]. Its 1+1-dimensional analogue was suggested by Mignemi in [72].

\subsection{Canonical Variables}

Now we will introduce a new set of phase space variables which obey canonical Poisson brackets are transform in the conventional way under SR Lorentz transformation. Somewhat similar considerations in parts have appeared before in [72 but exhaustive study of the full canonical phase space was given in [36]. Indeed, these variables are composites of phase space coordinates will have to suitable ordered upon quantization. But, in the classical framework they will prove to be very convenient and they drastically simplify the computations while analyzing phenomenological consequences of the modified Lorentz transformations. We will return to the quantum case at the end.

The two invariant quantities that we derived in (39,55) suggest the forms of these canonical avatars:

$$
X_{\mu} \equiv x_{\mu}\left(1-\frac{(\eta p)}{\kappa}\right)=x_{\mu}\left(1-\frac{E}{\kappa}\right) ; \quad P_{\mu} \equiv \frac{p_{\mu}}{\left(1-\frac{(\eta p)}{\kappa}\right)}=\frac{p_{\mu}}{\left(1-\frac{E}{\kappa}\right)} .
$$

We remind that the variables on the right hand side obey $\kappa$-LT laws. Using the NC algebra (42) it is easy to check the following:

$$
\left\{X_{\mu}, P_{\nu}\right\}=-g_{\mu \nu} ; \quad\left\{X_{\mu}, X_{\nu}\right\}=\left\{P_{\mu}, P_{\nu}\right\}=0 .
$$

Hence the $X, P$ phase space is canonical. The above relations in (56) are invertible,

$$
x_{\mu}=X_{\mu}\left(1+\frac{(\eta P)}{\kappa}\right)=X_{\mu}\left(1+\frac{P_{0}}{\kappa}\right) ; \quad p_{\mu}=\frac{P_{\mu}}{\left(1+\frac{(\eta P)}{\kappa}\right)}=\frac{P_{\mu}}{\left(1+\frac{P_{0}}{\kappa}\right)} .
$$

Next we consider Lorentz transformations of the canonical variables and find, for example,

$$
\begin{gathered}
T^{\prime}=t^{\prime}\left(1-\frac{E}{\kappa}\right)^{\prime}=\gamma \bar{\alpha}(t-v x)\left[1-\frac{\gamma}{\kappa \bar{\alpha}}\left(E-v p_{x}\right)\right] \\
=\gamma\left[t\left(1-\frac{E}{\kappa}\right)-v x\left(1-\frac{E}{\kappa}\right)\right]=\gamma(T-v X), \\
P_{0}^{\prime}=\frac{E^{\prime}}{\left(1-\frac{E}{\kappa}\right)^{\prime}}=\gamma\left(P_{0}-v P_{x}\right) .
\end{gathered}
$$

where $\bar{\alpha}=\alpha(-v)$ and we have used the identity $(\bar{\alpha})^{-1}=\alpha^{\prime}$. One can easily check that all the canonical variables $X_{\mu}, P_{\mu}$ obey SR LTs. 
Before putting to use this canonical variables we should add a cautionary remark. This mapping has led to conjectures [76] that in DSR the NC phase space formulation is redundant. However this mapping is classical and the quantization of this mapping will require a proper representation of the NC operators and this is an open problem. Some aspects have appeared in [52, 77] in the context of $\kappa$-Minkowski quantum field theory. Furthermore the NC quantum theories are qualitatively different with an inherent non-locality that obviously can not be captured in the canonical set up which, however, provides a very convenient framework to construct the quantum theory. An explicit example is given [78] where we construct the DSR generalization of Dirac fermions in a very simple way as compared to the original derivation [79].

\subsection{Deformed Symmetry Generators}

Let us show how to exploit the classical phase space in constructing deformed symmetry generators [36] (for the Snyder algebra see [38]). In the conventional case, the phase space algebraic structure of the point particle is invariant under the following symmetry transformations: translation, Lorentz rotation, dilation and special conformal transformation. On the other hand, the particle dispersion relation $P^{2}-m^{2}=0$ enjoys invariance under translation and Lorentz rotation, and the mass term $m$ breaks the symmetry under dilation and special conformal transformation. Finally, the symmetry generators satisfy a closed algebra among themselves.

In the $\kappa$-particle model our aim is to construct the generators in the $\kappa$-NC space that preserve invariances of both the $\kappa$-NC phase space algebra (42) and the structure of the algebra among generators (see below in (60) ). Then we will check how the $\kappa$-modified dispersion relation (MS relation (39) in the present case) is affected. Once again the canonical $\left(X_{\mu}, P_{\mu}\right)$ variables will do the trick. The idea is to first write down the generators in terms of $\left(X_{\mu}, P_{\mu}\right)$ degrees of freedom using the conventional form of the generators, (i.e. that of normal particle in normal phase space). They will obviously satisfy the standard closed algebra among generators:

$$
\begin{gathered}
\left\{J^{\mu \nu}, J^{\alpha \beta}\right\}=g^{\mu \beta} J^{\nu \alpha}+g^{\mu \alpha} J^{\beta \nu}+g^{\nu \beta} J^{\alpha \mu}+g^{\nu \alpha} J^{\mu \beta} ;\left\{J^{\mu \nu}, T^{\sigma}\right\}=g^{\nu \sigma} T^{\mu}-g^{\mu \sigma} T^{\nu} ; \\
\left\{J^{\mu \nu}, D\right\}=0 ;\left\{J^{\mu \nu}, K^{\sigma}\right\}=2 D\left(g^{\nu \sigma} X^{\mu}-g^{\mu \sigma} X^{\nu}\right)-X^{2}\left(g^{\nu \sigma} T^{\mu}-g^{\mu \sigma} T^{\nu}\right) \\
\left\{T^{\mu}, T^{\nu}\right\}=0 ;\left\{T^{\mu}, D\right\}=T^{\mu} ;\left\{T^{\mu}, K^{\nu}\right\}=2 D g^{\mu \nu}-2 J^{\mu \nu} \\
\{D, D\}=0 ;\left\{D, K^{\mu}\right\}=K^{\mu} ;\left\{K^{\mu}, K^{\nu}\right\}=0
\end{gathered}
$$

where $J_{\mu \nu}, T_{\mu}, D$ and $K_{\mu}$ stand for generators of Lorentz rotation, translation, dilation and special conformal transformation respectively. Their structures are given by,

$$
\begin{gathered}
J_{\mu \nu}=X_{\mu} P_{\nu}-X_{\nu} P_{\mu} ; T_{\mu}=P_{\mu} ; \quad D=(X P) ; \\
K_{\mu}=2(X P) X_{\mu}-X^{2} P_{\mu} .
\end{gathered}
$$

We exploit the map $\left(X_{\mu}, P_{\mu}\right) \rightarrow\left(x_{\mu}, p_{\mu}\right)$ given in (56) $)$ to rewrite the generators in the $\kappa$-NC spacetime:

$$
j_{\mu \nu}=x_{\mu} p_{\nu}-x_{\nu} p_{\mu} ; \quad t_{\mu}=\frac{p_{\mu}}{1-(\eta p) / \kappa} ; \quad ; \quad d=(x p) ;
$$




$$
k_{\mu}=(1-(\eta p) / \kappa)\left[2(x p) x_{\mu}-x^{2} p_{\mu}\right] .
$$

By construction, the generators in (62) will satisfy the same algebra (60) provided one uses the $\kappa$-NC algebra (42). These are the deformed generators. The infinitesimal transformation operators are,

$$
\begin{gathered}
j=\frac{1}{2} a^{\mu \nu} j_{\mu \nu}=\frac{1}{2} a^{\mu \nu}\left(x_{\mu} p_{\nu}-x_{\nu} p_{\mu}\right) ; \quad t=a^{\mu} t_{\mu}=\frac{(a p)}{1-(\eta p) / \kappa} ; d=a(x p) ; \\
k=a^{\mu} k_{\mu}=(1-(\eta p) / \kappa)\left[2(x p)(a x)-x^{2}(a p)\right],
\end{gathered}
$$

where generically $a$ denotes the infinitesimal parameter. Using the definition of small change in $A$ due to transformation $\delta_{b}$ as,

$$
\delta_{b} A=\left\{\delta_{b}, A\right\},
$$

Next we want to ascertain that the $\kappa$-NC algebra (42) is stable under the above symmetry operations. This is done by checking the validity of the identity,

$$
\{A, B\}=C \Rightarrow \delta_{b}\{A, B\}=\delta_{b} C,
$$

or more explicitly,

$$
\left\{\delta_{b} A, B\right\}+\left\{A, \delta_{b} B\right\}=\delta_{b} C .
$$

In the above we refer to (42) for $\{A, B\}=C$ and (62) for $\delta_{b}$. A straightforward but tedious calculation shows that the above identity is, indeed, valid. This assures us about the consistency of the whole procedure.

\subsection{Lagrangian for $\kappa$-Particle}

Lastly we will construct a Lagrangian for the $\kappa$-particle. This has been a topic of recent interest and several authors [80] have proposed models for particles with NC phase space of different structures. However, the model we propose here for $\kappa$ - $\mathrm{NC}$ phase space is quite elegant and can be expressed in a closed form.

Again the canonical variable approach becomes convenient since we are sure that the relativistic free particle action in terms of canonical $\left(X_{\mu}, P_{\mu}\right)$ degrees of freedom will be,

$$
L=\left(P^{\mu} \dot{X}_{\mu}\right)-\lambda\left(P^{2}-m^{2}\right) .
$$

We now convert this $L$ to a function depending on physical $\kappa$-NC phase space coordinates:

$$
\begin{aligned}
L & =\left(\frac{p^{\mu}}{1-\frac{(\eta p)}{\kappa}}\right)\left(x_{\mu}\left(1-\frac{(\eta p)}{\kappa}\right)\right) \cdot-\frac{\lambda}{2}\left(\frac{p^{2}}{\left(1-\frac{(\eta p)}{\kappa}\right)^{2}}-m^{2}\right) \\
& =(p \dot{x})-\frac{(p x)(\eta \dot{p})}{\kappa\left(1-\frac{(\eta p)}{\kappa}\right)}-\frac{\lambda}{2}\left(p^{2}-m^{2}\left(1-\frac{(\eta p)}{\kappa}\right)^{2}\right),
\end{aligned}
$$


where we have redefined the arbitrary multiplier $\lambda$. By the Hamiltonian constraint analysis, as formulated by Dirac [81] it is straightforward to check that the symplectic structure in (68) will induce the $\kappa$-NC phase space algebra and the $\lambda$-term will obviously impose the MS mass shell condition.

Finally, after eliminating the auxiliary variables we obtain the cherished Nambu-Goto Lagrangian for the $\kappa$-particle [36],

$$
L=\frac{m \sqrt{\dot{x}^{2}}}{\left(1+\frac{m(\eta \dot{x})}{\kappa \sqrt{\dot{x}^{2}}}\right)}\left(1+\frac{m}{\kappa}(\eta \dot{x})\left(\frac{(x \dot{x})}{\sqrt{\dot{x}^{2}}}\right) \cdot\right) .
$$

Notice that (69) is a higher derivative Lagrangian. Hamiltonian analysis of it will yield the $\kappa$-NC phase space algebra.

\section{Deformed and Twisted Gauge Symmetry in Noncom- mutative Field Theory:}

In this section we consider only canonical noncommutative space - a space where the noncommutative parameter $\theta^{\rho \sigma}$ is a real constant antisymmetric matrix. The functions defined on such a space satisfy the Moyal product (11). Replacement of functions by the spacetime coordinates in (11), gives

$$
\left[x^{\rho}, x^{\sigma}\right]_{*}=i \theta^{\rho \sigma} \text {. }
$$

This helps one to elevate a commutative field theory to a more general NC field theory by simply replacing the usual product by the $*$ product (11). Not surprisingly, the NC models defined on (70) violate the Lorentz invariance just like the algebra itself. However it has been shown that they are invariant under the twisted Poincare algebra 42], deformed with the Abelian twist element

$$
\mathcal{F}=\exp \left(\frac{i}{2} \theta^{\mu \nu} P_{\mu} P_{\nu}\right)
$$

where $P_{\mu}\left(=\partial_{\mu}\right)$ are the translation generators. Due to this twist, one can define the following multiplication map for the noncommutative (hatted) variables

$$
\mu \circ(\hat{\psi} \otimes \hat{\varphi})=\hat{\psi} \hat{\varphi} \rightarrow \mu_{*} \circ(\hat{\psi} \otimes \hat{\varphi})=\mu \circ \mathcal{F}^{-1}(\hat{\psi} \otimes \hat{\varphi}) \equiv \hat{\psi} * \hat{\varphi}
$$

which is precisely the ${ }^{*}$ product (11).

Gauge symmetries can be introduced in these NC models by the usual gauge invariance requirement. By construction these theories have the twisted Poincare symmetry and they are

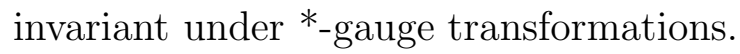

Recently an interesting study has been done by twisting not only the Poincare algebra but also the gauge algebra [82, 83]. An astonishing result was obtained -the NC theory turned out 
to be invariant under the commutative space gauge transformation provided the coproduct of the gauge generators are redefined by the same twist (171).

Thus, contrary to the commutative theory gauge symmetry in a noncommutative theory can be interpreted in two different ways. In one approach star deformed gauge transformations are taken, keeping the comultiplication (Leibniz) rule unchanged and in the other approach gauge transformations are taken as in the commutative case at the expense of a modified Leibniz rule. This rule is obtained from the usual Leibniz rule by the same twist operator used in defining the twisted Poincare generators. This shows a close correspondence between twisted Poincare symmetry and twisted gauge symmetry. In this review article we analyse both approaches within a common framework which is a generalization of the treatment of gauge symmetry in commutative space [84, 85, 86, 87, 88. We follow both the Lagrangian and Hamiltonian formulations which are complementary to each other.

An important point worth mentioning here is that while both types of gauge transformations keep the action invariant some controversies have been raised in the literature. In the case of star gauge transformation, gauge symmetries act only on the fields which is quite analogous to the commutative space theories. Whereas if ordinary gauge transformations with a twisted Leibniz rule is taken, then the transformations do not act only on the fields. Consequently, it is not clear whether the later type of gauge transformation can be considered as a physical symmetry or not. Discussions regarding this issue may be found in [89, 41].

\subsection{Lagrangian Analysis:}

The model we consider is the NC non-Abelian gauge field coupled with Dirac field?

$$
S=\int \mathrm{d}^{4} x\left[-\frac{1}{2} \operatorname{Tr}\left(\hat{F}_{\mu \nu}(x) * \hat{F}^{\mu \nu}(x)\right)+\hat{\bar{\psi}}(x) *\left(i \gamma^{\mu} D_{\mu} *-m\right) \hat{\psi}(x)\right] .
$$

where

$$
\begin{aligned}
D_{\mu} * \hat{\psi}(x) & \equiv \partial_{\mu}+i g \hat{A}_{\mu} * \hat{\psi}(x) \\
\hat{F}_{\mu \nu}(x) & \equiv \partial_{\mu} \hat{A}_{\nu}(x)-\partial_{\nu} \hat{A}_{\mu}(x)+i g\left[\hat{A}_{\mu}(x), \hat{A}_{\nu}(x)\right]_{*}
\end{aligned}
$$

The action (73) is invariant under both deformed gauge transformations [4],

$$
\begin{aligned}
& \delta \hat{A}_{\mu}=\mathcal{D}_{\mu} * \hat{\alpha}=\partial_{\mu} \hat{\alpha}+i g\left(\hat{A}_{\mu} * \hat{\alpha}-\hat{\alpha} * \hat{A}_{\mu}\right), \\
& \delta \hat{F}_{\mu \nu}=i g\left[\hat{F}_{\mu \nu}, \hat{\alpha}\right]_{*}=i g\left(\hat{F}_{\mu \nu} * \hat{\alpha}-\hat{\alpha} * \hat{F}_{\mu \nu}\right) \\
& \delta \hat{\psi}=-i g \hat{\alpha} * \hat{\psi} \\
& \delta \hat{\bar{\psi}}=i g \hat{\bar{\psi}} * \hat{\alpha}
\end{aligned}
$$

with the usual Leibniz Rule (LR),

$$
\delta(f * g)=(\delta f) * g+f *(\delta g)
$$

\footnotetext{
${ }^{3}$ Throughout this section we take $\theta^{0 i}=0$ to avoid higher order time derivatives.
} 
as well as the undeformed gauge transformations

$$
\begin{aligned}
& \delta_{\hat{\alpha}} \hat{A}_{\mu}=\mathcal{D}_{\mu} \hat{\alpha}=\partial_{\mu} \hat{\alpha}+i g\left(\hat{A}_{\mu} \hat{\alpha}-\hat{\alpha} \hat{A}_{\mu}\right), \\
& \delta_{\hat{\alpha}} \hat{F}_{\mu \nu}=i g\left[\hat{F}_{\mu \nu}, \hat{\alpha}\right]=i g\left(\hat{F}_{\mu \nu} \hat{\alpha}-\hat{\alpha} \hat{F}_{\mu \nu}\right) \\
& \delta_{\hat{\alpha}} \hat{\psi}=-i g \hat{\alpha} \hat{\psi} \\
& \delta_{\hat{\alpha}} \hat{\bar{\psi}}=i g \hat{\bar{\psi}} \hat{\alpha}
\end{aligned}
$$

with the twisted Leibniz Rule (TLR) [82, 83, 40],

$$
\begin{aligned}
\delta_{\hat{\alpha}}(f * g)= & \sum_{n}\left(\frac{-i}{2}\right)^{n} \frac{\theta^{\mu_{1} \nu_{1}} \cdots \theta^{\mu_{n} \nu_{n}}}{n !} \\
& \left(\delta_{\partial_{\mu_{1}} \cdots \partial_{\mu_{n}} \hat{\alpha}} f * \partial_{\nu_{1}} \cdots \partial_{\nu_{n}} g+\partial_{\mu_{1}} \cdots \partial_{\mu_{n}} f * \delta_{\partial_{\nu_{1}} \cdots \partial_{\nu_{n}} \hat{\alpha}} g\right) .
\end{aligned}
$$

We now elaborate on the derivation of the above rule (85), following Aschieri et. al. 83]. the *-product of two functions is defined in terms of a twist operator $\mathcal{F}_{\theta}\left(=e^{\frac{i}{2} \theta^{\rho \sigma} \partial_{\rho} \otimes \partial_{\sigma}}\right)$

$$
f * g=m_{0}\left\{\mathcal{F}_{\theta} f \otimes g\right\}
$$

where the map $m_{0}$ has been defined in (15). The twist operator can now be inverted to write the ordinary product as,

$$
f \cdot g=\left(\sum_{n=0}^{\infty}\left(-\frac{i}{2}\right)^{n} \frac{1}{n !} \theta^{\rho_{1} \sigma_{1}} \ldots \theta^{\rho_{n} \sigma_{n}}\left(\partial_{\rho_{1}} \ldots \partial_{\rho_{n}} f\right) * \partial_{\sigma_{1}}^{*} \ldots \partial_{\sigma_{n}}^{*} f\right) * g
$$

where $\partial_{\rho}^{*}$ is defined in the following way

$$
\begin{aligned}
\partial_{\rho}^{*}: & \partial_{\rho}^{*} f \equiv \partial_{\rho} f \\
& \partial_{\rho}^{*}(f * g)=\left(\partial_{\rho}^{*} f\right) * g+f *\left(\partial_{\rho}^{*} g\right)
\end{aligned}
$$

The equation (87) shows that $f \cdot g$ can be viewed as the *-action of a differential operator $X_{f}^{*}$ on $g$

$$
f \cdot g=X_{f}^{*} * g
$$

where

$$
X_{f}^{*}=\sum_{n=0}^{\infty}\left(-\frac{i}{2}\right)^{n} \frac{1}{n !} \theta^{\rho_{1} \sigma_{1}} \ldots \theta^{\rho_{n} \sigma_{n}}\left(\partial_{\rho_{1}} \ldots \partial_{\rho_{n}} f\right) * \partial_{\sigma_{1}}^{*} \ldots \partial_{\sigma_{n}}^{*} f
$$

Since the ${ }^{*}$-product is associative one can show that

$$
X_{f}^{*} * X_{g}^{*}=X_{f \cdot g}^{*}
$$

Now a gauge transformation

$$
\delta_{\alpha} \psi(x)=i \alpha \cdot \psi=i \alpha^{a}(x) T^{a} \psi(x)
$$


can be interpreted as a ${ }^{*}$-action

$$
\delta_{\alpha} \psi=i X_{\alpha^{a}}^{*} * T^{a} \psi=i X_{\alpha}^{*} * \psi=i \alpha \cdot \psi
$$

In a commutative space gauge theory the Hopf algebra

$$
\Delta \delta_{\alpha}(\phi \otimes \psi)=\left(\delta_{\alpha} \phi\right) \otimes \psi+\phi \otimes\left(\delta_{\alpha} \psi\right)
$$

together with the gauge transformations of the basic fields give the transformation of the product of fields

$$
\delta_{\alpha}(\phi \cdot \psi)=\delta_{\alpha} m_{0}(\phi \otimes \psi)=m_{0} \Delta \delta_{\alpha}(\phi \otimes \psi)
$$

In order to extend a Lie algebra to a Hopf algebra in noncommutative space the operator $\mathcal{F}_{\theta}^{-1}$ can be used in a convenient manner to get the following coproduct

$$
\Delta_{\theta}\left(\delta_{\hat{\alpha}}\right)(\phi \otimes \psi)=i \mathcal{F}_{\theta}^{-1}(\hat{\alpha} \otimes 1+1 \otimes \hat{\alpha}) \mathcal{F}_{\theta}(\phi \otimes \psi)
$$

which when acted by $m_{0}$ gives the expression (85).

The former (LR) formalism is probably more familiar and the latter (TLR) is a relatively new development [41, 42, 38]. In our analysis we take $\hat{A}_{\mu}$ and $\hat{F}_{\mu \nu}$ to be enveloping algebra valued, i.e. they are expanded over the basis $T^{a}$ which satisfy the Lie algebraic relation

$$
\left[T^{a}, T^{b}\right]=i f^{a b c} T^{c} ;\left\{T^{a}, T^{b}\right\}=d^{a b c} T^{c} .
$$

We also impose the trace condition

$$
\operatorname{Tr}\left(T^{a} T^{b}\right)=\frac{1}{2} \delta^{a b}
$$

so that $f^{a b c}$ is completely antisymmetric and $d^{a b c}$ is completely symmetric.

Leibniz Rule ( $L R$ ) formalism: We start by discussing the LR formalism. Let us recall that if $L_{a}$ denotes the Euler derivatives for a general Lagrangian density $\mathcal{L}\left(q_{a}, \partial_{\mu} q_{a}\right.$, ), corresponding to each gauge symmetry of the Lagrangian an identity can be written in terms of $L_{a}$ as [84, 85, 90]

$$
\Lambda^{b}(\mathbf{z}, t)=\left[\sum_{s=0}^{n} \int \mathrm{d}^{3} \mathbf{x} \frac{\partial^{s}}{\partial t^{s}}\left(\rho_{(s)}^{a b}(x, z) L_{a}(\mathbf{x}, t)\right)\right]=0 .
$$

where gauge transformation of the fields are of the form

$$
\delta q^{a}(\mathbf{x}, t)=\sum_{s=0}^{n}(-1)^{s} \int \mathrm{d}^{3} \mathbf{z} \frac{\partial^{s} \alpha^{b}(\mathbf{z}, t)}{\partial t^{s}} \rho_{(s)}^{a b}(x, z)
$$

with $\alpha$ and $\rho$ being the parameter and generator respectively, of the transformation. 
We show explicitly how to derive the deformed gauge transformation rules (76-79). The equations of motion for the action (73) can be obtained by setting Euler derivatives to zero. These are given by

$$
\begin{aligned}
& L^{\mu a}=-\left(\mathcal{D}_{\sigma} * \hat{F}^{\sigma \mu}\right)^{a}-g \hat{\psi}_{j}\left(\gamma^{\mu} T^{a}\right)_{i j} * \hat{\bar{\psi}}_{i} \\
& L_{i}=-i \partial_{\mu} \hat{\bar{\psi}}_{j}\left(\gamma^{\mu}\right)_{j i}-g \hat{\bar{\psi}}_{j} *\left(\gamma^{\mu} \hat{A}_{\mu}^{a} T^{a}\right)_{j i}-m \hat{\bar{\psi}}_{i} \\
& L_{i}^{\prime}=-i\left(\gamma^{\mu}\right)_{i j} \partial_{\mu} \hat{\psi}_{j}+g\left(\gamma^{\mu} \hat{A}_{\mu}^{a} T^{a}\right)_{i j} * \hat{\psi}_{j}+m \hat{\psi}_{i} .
\end{aligned}
$$

Here the noncommutative covariant derivative $\mathcal{D} *$ is defined in the adjoint representation (76)). The gauge identity for this system is [38, 39]

$$
\Lambda^{a} \equiv-\left(\mathcal{D}^{\mu} * L_{\mu}\right)^{a}-i g T_{i j}^{a} \hat{\psi}_{j} * L_{i}-i g T_{j i}^{a} L_{i}^{\prime} * \hat{\bar{\psi}}_{j}=0 .
$$

Comparing (105) and (100) different gauge generators can be obtained. As an example,

$$
\left.\Lambda^{a}\right|_{L_{i}}=\frac{g}{2} f^{a b c}\left\{\hat{A}^{i b}, L_{i}^{c}\right\}_{*}-i \frac{g}{2} d^{a b c}\left[\hat{A}^{i b}, L_{i}^{c}\right]_{*}-\partial^{i z} L_{i}^{a} .
$$

Using the properties

$$
\int \mathrm{d}^{4} x A(x) * B(x)=\int \mathrm{d}^{4} x A(x) B(x)=\int \mathrm{d}^{4} x B(x) * A(x)
$$

and

$$
\int \mathrm{d}^{4} x(A * B * C)=\int \mathrm{d}^{4} x(B * C * A)=\int \mathrm{d}^{4} x(C * A * B)
$$

(106) is written in the following way

$$
\begin{aligned}
& \left.\Lambda^{a}\right|_{L_{i}}(\mathbf{z}, t) \\
= & -\int \mathrm{d}^{3} \mathbf{x} \frac{g}{2}\left(f^{a b c}\left\{\delta^{3}(\mathbf{x}-\mathbf{z}), \hat{A}^{i c}(x)\right\}_{*}+i d^{a b c}\left[\delta^{3}(\mathbf{x}-\mathbf{z}), \hat{A}^{i c}(x)\right]_{*}\right) * L_{i}^{b}(x) \\
& -\int \mathrm{d}^{3} \mathbf{x} \delta^{a b} \partial^{i \mathbf{z}} \delta^{3}(\mathbf{x}-\mathbf{z}) L_{i}^{b}(x) .
\end{aligned}
$$

Comparing with (100) we obtain,

$$
\begin{aligned}
\rho_{(0)}^{b i a}(x, z)= & -\delta^{a b} \partial^{i \mathbf{z}} \delta^{3}(\mathbf{x}-\mathbf{z})- \\
& \frac{g}{2} f^{a b c}\left\{\delta^{3}(\mathbf{x}-\mathbf{z}), \hat{A}^{i c}(x)\right\}_{*}-i \frac{g}{2} d^{a b c}\left[\delta^{3}(\mathbf{x}-\mathbf{z}), \hat{A}^{i c}(x)\right]_{*} .
\end{aligned}
$$

Other components of the gauge generator can be obtained in a similar way. These are

$$
\begin{aligned}
\rho_{(0)}^{b 0 a}(x, z) & =-\frac{g}{2} f^{a b c}\left\{\delta^{3}(\mathbf{x}-\mathbf{z}), \hat{A}_{0}^{c}(x)\right\}_{*}-i \frac{g}{2} d^{a b c}\left[\delta^{3}(\mathbf{x}-\mathbf{z}), \hat{A}_{0}^{c}(x)\right]_{*} \\
\rho_{(1)}^{b 0 a}(x, z) & =-\delta^{a b} \delta^{3}(\mathbf{x}-\mathbf{z}) \\
\phi_{i(0)}^{a}(x, z) & =-i g T_{i j}^{a} \delta^{3}(\mathbf{x}-\mathbf{z}) * \hat{\psi}_{j}(x) \\
\phi_{i(0)}^{\prime a}(x, z) & =-i g T_{j i}^{a} \hat{\bar{\psi}}_{j}(x) * \delta^{3}(\mathbf{x}-\mathbf{z})
\end{aligned}
$$


Let us next consider the gauge transformations. From (101) we write the gauge transformation equation for the space component of the gauge field

$$
\begin{aligned}
\delta \hat{A}^{i a}(\mathbf{x}, t) & =\sum_{s}(-1)^{s} \int \mathrm{d}^{3} \mathbf{z} \frac{\partial^{s} \hat{\alpha}^{b}(\mathbf{z}, t)}{\partial t^{s}} * \rho_{(s)}^{a i b}(x, z) \\
& =\int \mathrm{d}^{3} \mathbf{z}\left(\hat{\alpha}^{b}(\mathbf{z}, t) * \rho_{(0)}^{a i b}(x, z)\right)
\end{aligned}
$$

Exploiting the identity [91, 38, 39]

$$
A(x) * \delta(x-z)=\delta(x-z) * A(z)
$$

and interchanging $a, b$, the generator (110) is recast as,

$$
\begin{aligned}
\rho_{(0)}^{a i b}(x, z)= & -\delta^{a b} \partial^{i \mathbf{z}} \delta^{3}(\mathbf{x}-\mathbf{z})+ \\
& \frac{g}{2} f^{a b c}\left\{\delta^{3}(\mathbf{x}-\mathbf{z}), \hat{A}^{i c}(z)\right\}_{*}+i \frac{g}{2} d^{a b c}\left[\delta^{3}(\mathbf{x}-\mathbf{z}), \hat{A}^{i c}(z)\right]_{*}
\end{aligned}
$$

Use of (117) along with the identities (107) and (108) in (115) implies that

$$
\delta \hat{A}^{i a}=\partial^{i} \hat{\alpha}^{a}-\frac{g}{2} f^{a b c}\left\{\hat{A}^{i b}, \hat{\alpha}^{c}\right\}_{*}+i \frac{g}{2} d^{a b c}\left[\hat{A}^{i b}, \hat{\alpha}^{c}\right]_{*}=\left(\mathcal{D}^{i} * \hat{\alpha}\right)^{a}
$$

This is the space component of (76) . The time component can also be obtained in a similar manner 39].

Twisted Leibniz Rule TLR formalism: Now we show how the TRL appears. For simplicity we take the pure gauge theory

$$
S=-\frac{1}{2} \int \mathrm{d}^{4} x \operatorname{Tr}\left(\hat{F}_{\mu \nu}(x) * \hat{F}^{\mu \nu}(x)\right)
$$

Using the twisted gauge transformation (85)

$$
\delta_{\hat{\alpha}}\left(\hat{A}_{\mu} * \hat{A}_{\nu}\right)=\partial_{\mu} \hat{\alpha} \hat{A}_{\nu}+\hat{A}_{\mu} \partial_{\nu} \hat{\alpha}-i g\left[\hat{\alpha},\left(\hat{A}_{\mu} * \hat{A}_{\nu}\right)\right]
$$

the gauge transformation of the field strength tensor

$$
\begin{aligned}
\delta_{\hat{\alpha}} \hat{F}_{\mu \nu}= & \partial_{\mu} \delta_{\hat{\alpha}} \hat{A}_{\nu}-\partial_{\nu} \delta_{\hat{\alpha}} \hat{A}_{\nu}+i g \delta_{\hat{\alpha}}\left[\hat{A}_{\mu}, \hat{A}_{\nu}\right]_{*} \\
= & \partial_{\mu}\left(\partial_{\nu} \hat{\alpha}+i g\left[\hat{A}_{\nu}, \hat{\alpha}\right]\right)-\partial_{\nu}\left(\partial_{\mu} \hat{\alpha}+i g\left[\hat{A}_{\mu}, \hat{\alpha}\right]\right) \\
& +i g\left(\left[\partial_{\mu} \hat{\alpha}, \hat{A}_{\nu}\right]+\left[\hat{A}_{\mu}, \partial_{\nu} \hat{\alpha}\right]-i g\left[\hat{\alpha},\left[\hat{A}_{\mu}, \hat{A}_{\nu}\right]_{*}\right]\right) \\
= & -i g\left[\hat{\alpha}, \hat{F}_{\mu \nu}\right] .
\end{aligned}
$$

Likewise one finds,

$$
\delta_{\hat{\alpha}}\left(\hat{F}^{\mu \nu} * \hat{F}_{\mu \nu}\right)=-i g\left[\hat{\alpha}, \hat{F}^{\mu \nu} * \hat{F}_{\mu \nu}\right]
$$


Both $\hat{F}_{\mu \nu}$ and $\hat{F}_{\mu \nu} * \hat{F}^{\mu \nu}$ have the usual (undeformed) transformation properties. Thus the action (119) is invariant under the gauge transformation (120) and the deformed coproduct rule $(\underline{85})$.

There is another way of interpreting the gauge invariance which makes contact with the gauge identity.

Making a gauge variation of the action (119) and taking into account the twisted coproduct rule (85), we get

$$
\begin{aligned}
\delta_{\hat{\alpha}} S= & -\frac{1}{2} \int \mathrm{d}^{4} x \operatorname{Tr} \delta_{\hat{\alpha}}\left(\hat{F}_{\mu \nu} * \hat{F}^{\mu \nu}\right) \\
= & -\frac{1}{2} \int \mathrm{d}^{4} x\left[\operatorname { T r } \left(\delta_{\hat{\alpha}} \hat{F}_{\mu \nu} * \hat{F}^{\mu \nu}+\hat{F}_{\mu \nu} * \delta_{\hat{\alpha}} \hat{F}^{\mu \nu}\right.\right. \\
& -\frac{i}{2} \theta^{\mu_{1} \nu_{1}}\left(\delta_{\partial_{\mu_{1}} \hat{\alpha}} \hat{F}_{\mu \nu} * \partial_{\nu_{1}} \hat{F}^{\mu \nu}+\partial_{\mu_{1}} \hat{F}_{\mu \nu} * \delta_{\partial_{\nu_{1}} \hat{\alpha}} \hat{F}^{\mu \nu}\right) \\
& +\cdot \cdot)] .
\end{aligned}
$$

Now using the result (122) each term of (126) can be computed separately. For example we concentrate on the first term. Using the identity (107) and the trace condition (99) we write the first term as

$$
\begin{aligned}
\left.\delta_{\hat{\alpha}} S\right|_{1 \text { st term }} & =-\frac{1}{4} \int \mathrm{d}^{4} x\left(\delta_{\hat{\alpha}} \hat{F}^{\mu \nu a} * \hat{F}_{\mu \nu}^{a}+\hat{F}^{\mu \nu a} * \delta_{\hat{\alpha}} \hat{F}_{\mu \nu}^{a}\right) \\
& =-\frac{1}{2} \int \mathrm{d}^{4} x \delta_{\hat{\alpha}} \hat{F}^{\mu \nu a} \hat{F}_{\mu \nu}^{a} .
\end{aligned}
$$

Making use of (122) and dropping the surface terms the above expression is found out to be,

$$
\begin{aligned}
\left.\delta_{\hat{\alpha}} S\right|_{1 \mathrm{st} \mathrm{term}}=-\int \mathrm{d}^{4} x \quad \hat{\alpha}^{a} & \left(-\partial^{\mu} \partial^{\nu} \hat{F}_{\mu \nu}-i g \partial^{\mu}\left[\hat{A}^{\nu}, \hat{F}_{\mu \nu}\right]-i g\left[\hat{A}^{\mu}, \partial^{\nu} \hat{F}_{\mu \nu}\right]\right. \\
& \left.+g^{2}\left[\hat{A}^{\mu} * \hat{A}^{\nu}, \hat{F}_{\mu \nu}\right]\right)^{a} .
\end{aligned}
$$

The second term of (126) is identically zero due to the antisymmetric nature of $\theta^{\mu \nu}$. We write that as,

$$
\begin{aligned}
\left.\delta_{\hat{\alpha}} S\right|_{2 \text { nd term }}= & -\frac{1}{2} \int \mathrm{d}^{4} x \hat{\alpha}^{a} \frac{i}{2} \theta^{\mu_{1} \nu_{1}}\left(-i g\left\{\partial_{\mu_{1}} \hat{F}^{\mu \nu}, \partial_{\nu_{1}} \hat{F}_{\mu \nu}\right\}\right)^{a} \\
= & -\int \mathrm{d}^{4} x \hat{\alpha}^{a} \frac{i}{2} \theta^{\mu_{1} \nu_{1}}\left(-i g\left\{\partial_{\mu_{1}} \partial^{\mu} \hat{A}^{\nu}, \partial_{\nu_{1}} \hat{F}_{\mu \nu}\right\}\right. \\
& \left.+g^{2}\left\{\partial_{\mu_{1}}\left(\hat{A}^{\mu} * \hat{A}^{\nu}\right), \partial_{\nu_{1}} \hat{F}_{\mu \nu}\right\}\right)^{a} \\
= & -\int \mathrm{d}^{4} x \hat{\alpha}^{a} \frac{i}{2} \theta^{\mu_{1} \nu_{1}}\left(-i g \partial^{\mu}\left\{\partial_{\mu_{1}} \hat{A}^{\nu}, \partial_{\nu_{1}} \hat{F}_{\mu \nu}\right\}-\right. \\
& \left.i g\left\{\partial_{\mu_{1}} \hat{A}^{\mu}, \partial_{\nu_{1}} \partial^{\nu} \hat{F}_{\mu \nu}\right\}+g^{2}\left\{\partial_{\mu_{1}}\left(\hat{A}^{\mu} * \hat{A}^{\nu}\right), \partial_{\nu_{1}} \hat{F}_{\mu \nu}\right\}\right)^{a} .
\end{aligned}
$$


Other terms can be obtained in a similar manner. Combining all these terms we finally get,

$$
\begin{aligned}
\delta_{\hat{\alpha}} S= & -\int \mathrm{d}^{4} x \hat{\alpha}^{a}\left(-\partial^{\mu} \partial^{\nu} \hat{F}_{\mu \nu}-i g \partial^{\mu}\left[\hat{A}^{\nu}, \hat{F}_{\mu \nu}\right]_{*}-i g\left[\hat{A}^{\mu}, \partial^{\nu} \hat{F}_{\mu \nu}\right]_{*}\right. \\
& \left.+g^{2}\left[\hat{A}^{\mu} * \hat{A}^{\nu}, \hat{F}_{\mu \nu}\right]_{*}\right)^{a} \\
= & -\int \mathrm{d}^{4} x \hat{\alpha}^{a} \Lambda^{a}
\end{aligned}
$$

where,

$$
\Lambda^{a}=-\left(\mathcal{D}^{\mu} * L_{\mu}\right)^{a}=-\left(\mathcal{D}^{\mu} * \mathcal{D}^{\sigma} * \hat{F}_{\sigma \mu}\right)^{a}
$$

that vanishes identically. Note that this is exactly the same as the expression in the gauge identity (105) without the fermionic fields. This proves the invariance of the action.

Let us now repeat the analysis of the previous section for TLR. Since the gauge transformations are undeformed, the gauge generators are expected to have the same form as in the commutative space. To see this note that the gauge variation of the zeroth component of the $\hat{A}_{\mu}$ field, following from (181), can be written as,

$$
\begin{aligned}
\delta_{\hat{\alpha}} \hat{A}_{0}^{a}(z) & =\partial_{0} \hat{\alpha}^{a}(z)-g f^{a b c} \hat{A}_{0}^{b}(z) \hat{\alpha}^{c}(z) \\
& =g \int \mathrm{d}^{3} \mathbf{z} f^{a b c} \hat{A}_{0}^{c} \hat{\alpha}^{b} \delta^{3}(\mathbf{x}-\mathbf{z})+\int \mathrm{d}^{3} \mathbf{z} \delta^{a b} \delta^{3}(\mathbf{x}-\mathbf{z}) \frac{\partial}{\partial t} \hat{\alpha}^{b}
\end{aligned}
$$

Clearly the above result can be expressed in our standard form (101),

$$
\begin{aligned}
\delta_{\hat{\alpha}} \hat{A}_{0}^{a}(z) & =\sum_{s}(-1)^{s} \int \mathrm{d}^{3} \mathbf{z} \frac{\partial^{s} \hat{\alpha}^{b}(\mathbf{z}, t)}{\partial t^{s}} \rho_{(s)}^{a 0 b}(x, z) \\
& =\int \mathrm{d}^{3} \mathbf{z} \hat{\alpha}^{b}(\mathbf{z}, t) \rho_{(0)}^{a 0 b}(x, z)-\int \mathrm{d}^{3} \mathbf{z} \frac{\partial \hat{\alpha}^{b}(\mathbf{z}, t)}{\partial t} \rho_{(1)}^{a 0 b}(x, z)
\end{aligned}
$$

where

$$
\begin{aligned}
& \rho_{(0)}^{a 0 b}(x, z)=g f^{a b c} \hat{A}_{0}^{c} \delta^{3}(\mathbf{x}-\mathbf{z}) \\
& \rho_{(1)}^{a 0 b}(x, z)=-\delta^{a b} \delta^{3}(\mathbf{x}-\mathbf{z})
\end{aligned}
$$

is the gauge generator. Similarly from the space part of (81) we find

$$
\rho_{(0)}^{a i b}(x, z)=-\delta^{a b} \partial^{i \mathbf{z}} \delta^{3}(\mathbf{x}-\mathbf{z})+g f^{a b c} \hat{A}_{i}^{c} \delta^{3}(\mathbf{x}-\mathbf{z}) .
$$

Now as already implied in (135), there is a gauge identity for this system,

$$
\Lambda^{a}=-\left(\mathcal{D}^{\mu} * L_{\mu}\right)^{a}=0
$$

where $L_{\mu}$ is the Euler derivative defined in (135). The gauge identity and the Euler derivatives are mapped by the relation [38, 39],

$$
\Lambda^{a}(\mathbf{z}, t)=\sum_{s=0}^{n} \int \mathrm{d}^{3} \mathbf{x} \frac{\partial^{s}}{\partial t^{s}}\left(\rho_{(s)}^{\prime b \mu a}(x, z) L_{\mu}^{b}(\mathbf{x}, t)\right)
$$


where the values of $\rho_{(0)}^{\prime b \mu a}(x, z)$ and $\rho_{(1)}^{\prime b \mu a}(x, z)$ are equal to those of $\rho_{(0)}^{b \mu a}$ and $\rho_{(1)}^{b \mu a}$ of the previous example, given in (111), (112) and (110). This happens since the Euler derivatives and the gauge identity are identical to those discussed above. Now we express $\rho^{\prime}$ in terms of $\rho$. To do this, (67) is rewritten under the identification $\rho=\rho^{\prime}$ as,

$$
\rho_{(0)}^{\prime b 0 a}(x, z)=-\frac{g}{2} f^{a b c}\left\{\delta^{3}(\mathbf{x}-\mathbf{z}), \hat{A}_{0}^{c}(x)\right\}_{*}-i \frac{g}{2} d^{a b c}\left[\delta^{3}(\mathbf{x}-\mathbf{z}), \hat{A}_{0}^{c}(x)\right]_{*} .
$$

Now making use of the definition of star product, the above expression is written in the following way

$$
\begin{aligned}
\rho_{(0)}^{\prime b 0 a}(x, z)= & -g f^{a b c} \hat{A}_{0}^{c} \delta^{3}(\mathbf{x}-\mathbf{z})-g \sum_{n=1}^{\infty}\left(\frac{i}{2}\right)^{n} \frac{\theta^{\mu_{1} \nu_{1}} \cdots \theta^{\mu_{n} \nu_{n}}}{n !} \\
& {\left[\left(\frac{f^{a b c}}{2}+i \frac{d^{a b c}}{2}\right) \partial_{\mu_{1}} \cdots \partial_{\mu_{n}} \delta^{3}(\mathbf{x}-\mathbf{z}) \partial_{\nu_{1}} \cdots \partial_{\nu_{n}} \hat{A}^{0 c}(x)\right.} \\
& \left.\left(+\frac{f^{a b c}}{2}-i \frac{d^{a b c}}{2}\right) \partial_{\mu_{1}} \cdots \partial_{\mu_{n}} \hat{A}^{0 c}(x) \partial_{\nu_{1}} \cdots \partial_{\nu_{n}} \delta^{3}(\mathbf{x}-\mathbf{z})\right] .
\end{aligned}
$$

Note that the $\theta$ independent term is nothing but the gauge generator $\rho_{(0)}^{b 0 a}\left(\right.$ or $\left.-\rho_{(0)}^{a 0 b}\right)$ given in (138). Similarly calculating the other components $\rho_{(0)}^{\prime b i a}$ and $\rho_{(1)}^{\prime b 0 a}$ from (112) and (110) we obtain,

$$
\begin{aligned}
\rho_{(0)}^{\prime b \mu a}(x, z)= & \rho_{(0)}^{b \mu a}(x, z)-g \sum_{n=1}^{\infty}\left(\frac{i}{2}\right)^{n} \frac{\theta^{\mu_{1} \nu_{1}} \cdots \theta^{\mu_{n} \nu_{n}}}{n !} \\
& {\left[\left(\frac{f^{a b c}}{2}+i \frac{d^{a b c}}{2}\right) \partial_{\mu_{1}} \cdots \partial_{\mu_{n}} \delta^{3}(\mathbf{x}-\mathbf{z}) \partial_{\nu_{1}} \cdots \partial_{\nu_{n}} \hat{A}^{\mu c}(x)\right.} \\
& \left.\left(+\frac{f^{a b c}}{2}-i \frac{d^{a b c}}{2}\right) \partial_{\mu_{1}} \cdots \partial_{\mu_{n}} \hat{A}^{\mu c}(x) \partial_{\nu_{1}} \cdots \partial_{\nu_{n}} \delta^{3}(\mathbf{x}-\mathbf{z})\right] \\
\rho_{(1)}^{\prime b 0 a}(x, z)= & \rho_{(1)}^{b 0 a}(x, z) .
\end{aligned}
$$

Here the generator of the system is $\rho$ (138,139,140). Although it remains undeformed, the relation mapping the gauge identity with the generator is twisted. The additional twisted terms are explicitly given in (145). In the commutative space limit the twisted terms naturally vanish.

\subsection{Hamiltonian Analysis}

We consider a system with a canonical Hamiltonian $H_{c}$ and a set of first class constraints $\Phi_{a} \approx 0$. In general $\Phi_{a}$ includes both the primary $\left(\Phi_{a_{1}}\right)$ and secondary constraints $\left(\Phi_{a_{2}}\right)$ and satisfy the following involutive Poisson algebra [84, 86, 87, 88, 92, 81]

$$
\begin{aligned}
& \left\{H_{c}, \Phi_{a}(x)\right\}=\int \mathrm{d} y V_{a}^{b}(x, y) \Phi_{b}(y) \\
& \left\{\Phi_{a}(x), \Phi_{b}(y)\right\}=\int \mathrm{d} z C_{a b}^{c}(x, y, z) \Phi_{c}(z)
\end{aligned}
$$


where $V$ and $C$ are structure functions. The gauge transformation of a variable $F$ is obtained from the Poisson bracket

$$
\delta F(x)=\int \mathrm{d} y\{F(x), G(y)\}
$$

where $G$ is the generator of the system. According to Dirac's algorithm it is a linear combination of all the first class constraints,

$$
G=\int \mathrm{d} x \epsilon^{a}(x) * \Phi_{a}(x) .
$$

Here the number of independent gauge parameter is $a_{1}$. Other parameters are fixed by the relation 86 , 87]

$$
\begin{aligned}
\frac{\mathrm{d} \epsilon^{b_{2}}(x)}{\mathrm{d} t}= & \int \mathrm{d} y \epsilon^{a}(y) V_{a}^{b_{2}}(y, x) \\
& +\int \mathrm{d} y \mathrm{~d} z \epsilon^{a}(y) v^{a_{1}}(z) C_{a_{1} a}^{b_{2}}(z, y, x)
\end{aligned}
$$

LR formalism: The general analysis discussed above is now used here for the model (73) to study its Hamiltonian description. Due to the presence of grassmanian variables in our model (73), the Poisson brackets should be replaced by the graded bracket: 4 . The canonical momenta of the Lagrangian (73),

$$
\hat{\pi}_{\sigma}^{a}=\frac{\partial \mathcal{L}}{\partial \dot{\hat{A}}^{\sigma a}}=\hat{F}_{\sigma 0}^{a}
$$

satisfy the basic Poisson bracket relation

$$
\left\{\hat{A}^{\mu a}(x), \hat{\pi}_{\nu}^{b}(y)\right\}=\delta^{a b} \delta_{\nu}^{\mu} \delta(x-y)
$$

The zeroth component of the momenta (152) leads to a primary constraint

$$
\Phi_{1}^{a}=\hat{\pi}_{0}^{a} \approx 0 .
$$

The canonical Hamiltonian of the system is given by,

$$
\begin{aligned}
H= & \int \mathrm{d} x\left[\frac{1}{2} \hat{\pi}^{i c} * \hat{\pi}^{i c}+\frac{1}{4} \hat{F}_{i j}^{a} * \hat{F}^{i j a}-\left(\mathcal{D}_{i} * \hat{\pi}^{i}\right)^{a} * \hat{A}_{0}^{a}\right. \\
& \left.-i \hat{\bar{\psi}} * \gamma^{i} \partial_{i} \hat{\psi}+g \hat{\bar{\psi}} * \gamma^{\mu} \hat{A}_{\mu} * \hat{\psi}+m \hat{\bar{\psi}} * \hat{\psi}\right]
\end{aligned}
$$

Now using (153), the secondary constraints of the system are computed

$$
\Phi_{2}^{a}=\left\{H, \Phi_{1}^{a}\right\}=\left\{H, \hat{\pi}_{0}^{a}\right\}=\left(\mathcal{D}_{i} * \hat{\pi}_{i}\right)^{a}-g \hat{\psi}_{\lambda} *\left(T^{a}\right)_{\sigma \lambda}\left(\hat{\psi}^{\dagger}\right)_{\sigma} \approx 0 .
$$

\footnotetext{
${ }^{4}$ For fermions $\left\{\hat{\psi}_{\alpha}(x), \hat{\psi}_{\beta}^{\dagger}(y)\right\}=-i \delta_{\alpha \beta} \delta(x-y)$.
} 
Note that this constraint is the zeroth component of the equation of motion of the gauge field expressed in phase space variables. The constraint algebra found is [91]

$$
\left\{\Phi_{2}^{a}(x), \Phi_{2}^{b}(y)\right\}=\frac{g}{2} f^{a b c}\left\{\delta(x-y), \Phi_{2}^{c}(x)\right\}_{*}-i \frac{g}{2} d^{a b c}\left[\delta(x-y), \Phi_{2}^{c}(x)\right]_{*} .
$$

All other brackets are zero. The involutive algebra of the canonical Hamiltonian with the constraints is found to be,

$$
\begin{aligned}
& \left\{H_{c}, \Phi_{1}^{a}\right\}=\Phi_{2}^{a} \\
& \left\{H_{c}, \Phi_{2}^{a}\right\}=-\frac{g}{2} f^{a b c}\left\{\hat{A}^{0 b}, \Phi_{2}^{c}\right\}_{*}+i \frac{g}{2} d^{a b c}\left[\hat{A}^{0 b}, \Phi_{2}^{c}\right]_{*} .
\end{aligned}
$$

The $V$ function defined in (147) is found from the algebra (158) and (159)

$$
\begin{aligned}
\left(V_{1}^{2}\right)^{a b}(x, y)= & \delta^{a b} \delta(x-y) \\
\left(V_{2}^{2}\right)^{a b}(x, y)= & \frac{g}{2} f^{a b c}\left\{\delta(x-y), \hat{A}^{0 c}(y)\right\}_{*} \\
& +i \frac{g}{2} d^{a b c}\left[\delta(x-y), \hat{A}^{0 c}(y)\right]_{*}
\end{aligned}
$$

Now the term $C_{a_{1} a}^{b_{2}}$ of (148) vanishes due to the constraint algebra. So from (151) we get $\epsilon^{1}=\left(\mathcal{D}_{0} * \epsilon^{2}\right)$ [38, 92]. Thus (150) is expressed in terms of the single parameter $\left(\epsilon^{2}\right)$ as,

$$
G=\int \mathrm{d} x\left(\mathcal{D}_{0} * \epsilon^{2}\right)^{a} * \Phi_{1}^{a}+\epsilon^{2 a} * \Phi_{2}^{a}
$$

where the constraints $\Phi_{1}$ and $\Phi_{2}$ were defined in (154) and (156). After obtaining the complete form of the generator, we can now calculate the variation of the different fields from (149),

Let us first study the gauge transformation of the field $\hat{A}^{\mu}$. The variation of its time component is

$$
\begin{aligned}
\delta \hat{A}_{0}^{a}(x) & =\int \mathrm{d} y\left(\mathcal{D}_{0} * \epsilon^{2}\right)^{b}(y) *\left\{\hat{A}_{0}^{a}(x), \hat{\pi}_{0}^{b}(y)\right\} \\
& =\int \mathrm{d} y\left(\mathcal{D}_{0} * \epsilon^{2}\right)^{b}(y) \delta^{a b} * \delta(x-y) \\
& =\left(\mathcal{D}_{0} * \epsilon^{2}\right)^{a}
\end{aligned}
$$

where we have used the identity (107). The variation of the space component is found to be $\delta \hat{A}_{i}^{a}(x)=\left(\mathcal{D}_{i} * \epsilon^{2}\right)^{a}(x)$. Combining this with (163) and identifying $\epsilon^{2} \rightarrow \hat{\alpha}$ we get (76). In a similar way (78) and (779) can be reproduced.

TLR formalism: So far we were discussing about the star deformed gauge transformation from a general Hamiltonian formulation which obeys the normal coproduct rule (80). But as discussed in the previous chapter the action (73) is also invariant under the undeformed gauge transformations (81, 84) with the twisted coproduct rule (85).

We now present an alternative interpretation of the twisted coproduct rule (85). The results (120) are seen to follow by using the standard coproduct rule (80) but pushing the gauge 
parameter $\hat{\alpha}$ outside the star operation at the end of the computations [38, 39]. Denoting this manipulation as,

$$
\delta_{\hat{\alpha}}(A * B) \sim\left(\delta_{\hat{\alpha}} A\right) * B+A *\left(\delta_{\hat{\alpha}} B\right)
$$

we find

$$
\begin{aligned}
\delta_{\hat{\alpha}}\left(\hat{A}_{\mu} * \hat{\psi}\right) & \sim\left(\delta_{\hat{\alpha}} \hat{A}_{\mu}\right) * \hat{\psi}+\hat{A}_{\mu} *\left(\delta_{\hat{\alpha}} \hat{\psi}\right) \\
& \sim\left(\partial_{\mu} \hat{\alpha}-i g \hat{\alpha}^{a}\left[T^{a}, \hat{A}_{\mu}\right]\right) * \hat{\psi}+\hat{A}_{\mu} *\left(-i g \hat{\alpha}^{a} T^{a} \hat{\psi}\right) \\
& =\partial_{\mu} \hat{\alpha} \hat{\psi}-i g \hat{\alpha}^{a}\left(\left[T^{a}, \hat{A}_{\mu}\right] * \hat{\psi}\right)-i g \hat{\alpha}^{a}\left(\hat{A}_{\mu} * T^{a} \hat{\psi}\right) \\
& =\partial_{\mu} \hat{\alpha} \hat{\psi}-i g \hat{\alpha}\left(\hat{A}_{\mu} * \hat{\psi}\right)
\end{aligned}
$$

which can also be obtained by using the twisted coproduct rule (85).

In order to obtain the undeformed gauge transformations (81, 84) and the relations (120) we give a prescription. In the computation of Poisson brackets, the gauge parameter has to be pushed outside the star operation at the end of the computations.

The gauge variation of the time component of $\hat{A}^{\mu}$ field is found by suitably Poisson bracketing with (162) (renaming $\epsilon^{2}$ as $\hat{\alpha}$ ),

$$
\begin{aligned}
\delta_{\hat{\alpha}} \hat{A}_{0}^{a}(x) & =\int \mathrm{d} y\left(\mathcal{D}_{0} * \hat{\alpha}\right)^{b}(y) *\left\{\hat{A}_{0}^{a}(x), \hat{\pi}_{0}^{b}(y)\right\} \\
& \sim \int \mathrm{d} y\left(\partial_{0} \hat{\alpha}^{a}-\frac{g}{2} f^{a b c}\left\{\hat{A}_{0}^{b}, \hat{\alpha}^{c}\right\}_{*}+i \frac{g}{2} d^{a b c}\left[\hat{A}_{0}^{b}, \hat{\alpha}^{c}\right]_{*}\right)(y) * \delta(x-y) \\
& =\partial_{0} \hat{\alpha}^{a}-g f^{a b c} \hat{A}_{0}^{b} \hat{\alpha}^{c}
\end{aligned}
$$

where in the last step we put $\hat{\alpha}$ outside the star product following our prescription. The variation of the space component is also calculated in a similar way

$$
\delta_{\hat{\alpha}} \hat{A}_{i}^{a}(x)=\partial_{i} \hat{\alpha}^{a}-g f^{a b c} \hat{A}_{i}^{b} \hat{\alpha}^{c}
$$

Combining (166) and (167) we write the gauge variation in a covariant notation

$$
\delta_{\hat{\alpha}} \hat{A}_{\mu}^{a}=\left(\mathcal{D}_{\mu} \hat{\alpha}\right)^{a} .
$$

The gauge variation of the fermionic field can be obtained in a similar way. The calculation of the gauge variation of composite fields needs some care. For example, consider the variation $\delta_{\hat{\alpha}}\left(\hat{A}_{\mu} * \hat{\psi}\right)$,

$$
\begin{aligned}
\delta_{\hat{\alpha}}\left(\hat{A}_{0}(x) * \hat{\psi}(x)\right)= & T^{a} \delta_{\hat{\alpha}}\left(\hat{A}_{0}^{a}(x) * \hat{\psi}(x)\right) \\
\sim & T^{a} \int \mathrm{d} y\left(\mathcal{D}_{0} * \hat{\alpha}^{a}\right)(y) * \delta(x-y) * \hat{\psi}(x) \\
& -i g T^{b} \int \mathrm{d} y \hat{\alpha}^{c}(y) * T^{c} \hat{\psi}(y) * \hat{A}_{0}^{b}(x) * \delta(x-y) .
\end{aligned}
$$


Using the identity (116) the argument of $\hat{\psi}$ and $\hat{A}_{0}^{b}$ is changed from $x$ to $y$ to obtain

$$
\begin{aligned}
\delta_{\hat{\alpha}}\left(\hat{A}_{0}(x) * \hat{\psi}(x)\right) \sim & T^{a} \int \mathrm{d} y\left(\mathcal{D}_{0} * \hat{\alpha}^{a}\right)(y) * \hat{\psi}(y) * \delta(x-y) \\
& -i g T^{b} \int \mathrm{d} y \hat{\alpha}^{c}(y) T^{c} * \hat{\psi}(y) * \delta(x-y) * \hat{A}_{0}^{b}(y) .
\end{aligned}
$$

Using the properties (107), (108) and finally removing the gauge parameter $\hat{\alpha}$ outside the star product we obtain

$$
\delta_{\hat{\alpha}}\left(\hat{A}_{0} * \hat{\psi}\right)=T^{a}\left(\partial_{0} \hat{\alpha}^{a} \hat{\psi}-g f^{a b c} \hat{\alpha}^{c}\left(\hat{A}_{0}^{b} * \hat{\psi}\right)\right)-i g T^{b} T^{c} \hat{\alpha}^{c}\left(\hat{A}_{0}^{b} * \hat{\psi}\right) .
$$

Using the symmetry algebra (98,99) it is easy to check that above result is the time component of the equation (120). similar result can also be found for the space part. The gauge variations of the other composites are computed in the same way reproducing the results (120) obtained by using the twisted coproduct rule.

\section{Noncommutative Gravity and Black Hole Physics}

The renewed interest in NC spacetime is mainly due to its relevance in quantum gravity research. Formulation of gravity theories over NC spacetime thus attracted a huge attention in the literature [93, 47]. There are various attempts to fit General theory of Relativity (GTR) in the context of NC space time. In [94] for example a deformation of Einstein's gravity was studied using a construction based on gauging the noncommutative $\mathrm{SO}(4,1)$ de Sitter group and the SW map [4] with subsequent contraction to $\operatorname{ISO}(3,1)$. Another construction of a noncommutative gravitational theory was proposed in [95]. Recently noncommutative gravity has been connected with stringy perspective [89]. In all these works the leading order noncommutative effects appear in the second order in the NC parameter $\theta$.

The introduction of non-commutativity spoils the symmetry under general coordinate transformation. However, the NC geometry is compatible with a restricted class of coordinate transformations which is volume preserving. The corresponding formulation of NC gravity [96], often referred to as the minimal theory, brings us to the realm of Unimodular Gravity [97. Initially, the leading order correction was reported to be linear in $\theta$ in this work. The model was reconsidered in [44] where it was shown by explicit construction that the first order correction actually vanishes. The second order corrections were later computed [98]. The noncommutative structure in [96, 44, 98] is constant $\theta_{\mu \nu}$. Subsequently, NC gravity in the minimal theory approach was developed with $\theta_{\mu \nu}$ having a Lie-algebraic structure [99]. The vanishing of the first order correction was again observed. The same phenomenon is observed in in calculations from various angles [100, 101]. Below we will show how the first order term vanishes by reviewing results from [99] in the Lie algebraic form of NC spacetime. The results for canonical noncommutativity [4] may be obtained from these by a limiting procedure.

The methodology of direct generalisation of gravity to NC spacetime that can yield results useful for different phenomenology is based on a perturbative expansion in the NC parameter 
$\theta$. The leading order $\mathrm{NC}$ correction being second order in the $\mathrm{NC}$ parameter is indeed small. However, this has been shown to have important phenomenological consequences [45] where NC generalization of the Schwarzschild solution has been worked out. Later on the impact of the NC effect on Charged black hole has been analysed [102, 103]. Below we will review the results of [102] where the deformed Reissner-Nordstrom solutions are given. The results for the Schwarzschild solutions given in [45] are obtainable by a limiting procedure from these results. Considering the complex steps involved in the computation of the $\mathrm{NC}$ corrections this correspondence is indeed notable.

The perturbative expansion employed in the NC gravity theories involve a cut off which removes the original nonlocality in the $\mathrm{NC}$ theories. This perturbative expansion is however an essential feature of incorporating $\mathrm{NC}$ effects in gravity. In the last few years an interesting study has been done in the literature [104, 10] where gravity is interpreted in a completely different manner within a noncommutative framework. Here gravity is not incorporated in the theory by hand rather it turns out to be an emergent structure from the electromagnetic phenomena in noncommutative spacetime. An alternative procedure is to consider the effect of the fuzziness in the level of the mean values [47, 105, 106. This "noncommutativity inspired" methodology has been pursued in [107]. We will conclude our review of NC gravity with a few results obtained in this approach.

\subsection{Lie Algebraic Noncommutative Gravity}

We consider noncommutativity of the form

$$
\begin{aligned}
{\left[\hat{x}^{\mu}, \hat{x}^{\nu}\right] } & =i \theta^{\mu \nu}(\hat{x}) \\
& =i \theta f_{\lambda}^{\mu \nu} \hat{x}^{\lambda}
\end{aligned}
$$

where $f_{\lambda}^{\mu \nu}$ are the structure constants. For consistency these constants assume a Lie algebraic structure so that $f_{\mu \nu \lambda}$ is antisymmetric in all the three indices.

The formulation of gravity on NC space time poses problems. This is seen by considering the general coordinate transformation,

$$
\hat{x}^{\mu} \rightarrow \hat{x}^{\prime \mu}=\hat{x}^{\mu}+\hat{\xi}^{\mu}(\hat{x})
$$

and realising that, for arbitrary $\hat{\xi}^{\mu}(\hat{x})$, it is not compatible with the algebra (172). However, as in the canonical case [96], it is possible to find a restricted class of coordinate transformations (173) which preserves the Lie - algebraic noncommutative algebra. We exploit the Weyl Wigner correspondence [1] to work in the deformed phase space with the ordinary multiplication substituted by the corresponding star product.

The noncommutative coordinates $\hat{x}^{\mu}$ satisfying (172) are the generators of an associative algebra $\mathcal{A}_{x}$. According to the Weyl correspondence we can associate an element of $\mathcal{A}_{x}$ with a function $f(x)$ of classical variables $x^{\mu}$ by the unique prescription. The ${ }^{*}$-product between two classical functions $f(x)$ and $g(x)$ is denoted by $f * g$ and is defined by the requirement functions 
with their product defined by the star product. When the generators satisfy the Lie structure the star product is explicitly given by [108, 109]

$$
f(x) * g(x)=\left.e^{\frac{i}{2} x^{\lambda} g_{\lambda}\left(i \frac{\partial}{\partial x^{\prime}}, i \frac{\partial}{\partial x^{\prime \prime}}\right)} f\left(x^{\prime}\right) g\left(x^{\prime \prime}\right)\right|_{\left(x^{\prime}, x^{\prime \prime}\right) \rightarrow x}
$$

where $g_{\lambda}$ is defined by,

$$
e^{i k_{\lambda} \hat{x}^{\lambda}} e^{i p_{\lambda} \hat{x}^{\lambda}}=e^{i\left\{k_{\lambda}+p_{\lambda}+\frac{1}{2} g_{\lambda}(k, p)\right\} \hat{x}^{\lambda}}
$$

The explicit form of $g_{\lambda}(k, p)$ is obtained as

$$
\begin{aligned}
g_{\lambda}(k, p)= & -\theta k_{\mu} p_{\nu} f_{\lambda}^{\mu \nu}+\frac{1}{6} \theta^{2} k_{\mu} p_{\nu}\left(p_{\sigma}-k_{\sigma}\right) f_{\delta}^{\mu \nu} f_{\lambda}^{\delta \sigma} \\
& +\frac{1}{24} \theta^{3}\left(p_{\sigma} k_{\beta}+k_{\sigma} p_{\beta}\right) k_{\mu} p_{\nu} f_{\delta}^{\mu \nu} f_{\alpha \sigma}^{\delta \sigma} f_{\lambda}^{\alpha \beta}+\ldots
\end{aligned}
$$

Now in order to preserve the noncommutative algebra (172) under the general coordinate transformation (173), $\xi^{\mu}$ must satisfy the condition,

$$
\hat{\xi}^{\mu}(x)=f_{\beta}^{\mu \alpha} x^{\beta} \partial_{\alpha} g(x)
$$

so that

$$
\left[\hat{x}^{\prime \mu}, \hat{x}^{\prime \nu}\right]=i \theta f_{\lambda}^{\mu \nu} \hat{x}^{\prime \lambda}
$$

Here the symbol $g(x)$ which appears in (177) denotes an arbitrary function. From (177) we find that

$$
\partial_{\mu} \hat{\xi}^{\mu}(x)=0
$$

which implies that the Jacobian of the transformations (173) is then unity. In other words the transformations are volume preserving. The corresponding theory thus belongs to the noncommutative version of unimodular gravity.

We have now all the tools at our disposal to develop the commutative equivalent theory of noncommutative gravity in the framework of Poincare gauge theory of gravity [93]. The corresponding noncommutative gauge transformation can be decomposed in the following way

$$
\hat{\Lambda}(\hat{x})=\hat{\xi}^{\mu}(\hat{x}) p_{\mu}+\frac{1}{2} \hat{\lambda}^{a b}(\hat{x}) \Sigma_{a b}
$$

Here $\hat{\xi}^{\mu}$ is the local translation of the tetrad which must be restricted to the form given in equation (177) in order to preserve the noncommutative algebra (172). The parameters $\hat{\lambda}^{a b}(\hat{x})$ characterize the local Lorentz transformations at $\hat{x}$ with $\Sigma_{a b}$ as the generators of the Lorentz group. In what follows we will assume the vector representation of $\operatorname{Sigma}_{a b}$. As is usual we will denote the general coordinates by the Greek indices and components with respect to the 
tetrad by Latin indices. Corresponding to the noncommutative gauge transformations (179) we introduce the gauge potential

$$
\hat{A}_{a}(\hat{x})=\left(\hat{D}_{a}\right)=i \hat{E}_{a}^{\mu}(\hat{x}) p_{\mu}+\frac{i}{2} \hat{\omega}_{a}^{b c}(\hat{x}) \Sigma_{b c}
$$

where $E_{a}^{\mu}(\hat{x})$ are the components of the noncommutative tetrad $\hat{E}_{a}$ which are also the gauge fields corresponding to general coordinate transformations and $\hat{\omega}_{a}^{b c}(\hat{x})$ are the spin connection fields associated with local Lorentz invariance. Since $p_{\mu}=-i \partial_{\mu}$, the noncommutative tetrad maps trivially on the commutative one [96]. Assuming the gauge transformations and the spin connection fields in the enveloping algebra approach [109] we can write the order $\theta$ corrections to these potentials. Using these, the field strength tensor is worked out. This is identified with the NC Riemann tensor under zero torsion. From the NC Riemann tensor the NC Ricci Scalar is straightforwardly computed. Thus we can write

$$
\hat{R}_{a b}=R_{a b}+R_{a b}^{(1)}+\mathcal{O}\left(\theta^{2}\right)
$$

where the correction term is obtained as,

$$
\begin{aligned}
R_{a b}^{(1)}= & \frac{1}{2} \theta^{c d}\left\{R_{a c}, R_{b d}\right\}-\frac{1}{4} \theta^{c d}\left\{\omega_{c},\left(\partial_{d}+\mathcal{D}_{d}\right) R_{a b}\right\} \\
& +\frac{1}{2} \theta_{b c} \theta^{d e} \partial_{e} \theta^{f c}\left\{R_{a f}, \omega_{d}\right\}-\frac{1}{2} \theta_{a c} \theta^{d e} \partial_{e} \theta^{f c}\left\{R_{b f}, \omega_{d}\right\} .
\end{aligned}
$$

The Ricci tensor $\hat{R}_{a}^{c}=\hat{R}_{a b}{ }^{b c}$ and the Ricci scalar $\hat{R}=\hat{R}_{a b}{ }^{a b}$ are formed to construct the action

$$
\begin{aligned}
S & =\int d^{4} x \frac{1}{2 \kappa^{2}} \hat{R}(\hat{x}) \\
& =\int d^{4} x \frac{1}{2 \kappa^{2}}\left(R(x)+R^{(1)}(x)\right)+\mathcal{O}\left(\theta^{2}\right) .
\end{aligned}
$$

The first order correction term to the Lagrangian is

$$
R^{(1)}(x)=R_{a b}^{(1) a b}=\left[R_{a b}^{(1)}\right]^{a b}
$$

It is convenient to arrange the correction as

$$
\left[R_{a b}^{(1)}\right]^{a b}=\mathcal{R}_{1}+\mathcal{R}_{2}+\mathcal{R}_{3}+\mathcal{R}_{4}
$$

where $\mathcal{R}_{1}, \ldots, \mathcal{R}_{4}$ correspond to the contributions coming from the four pieces appearing on the right hand side of (182) in the same order. Exploiting the various symmetries of the Riemann Tensor, spin connection and the noncommutative structure $\theta^{a b}$ we can easily show that both $\mathcal{R}_{1}$ and $\mathcal{R}_{2}$ individually vanish. Note that these terms do not depend on the coordinate dependence of $\theta^{a b}$ and will remain valid for canonical noncommutative structure. This is the canonical limit of [96, 44]. The last two terms on the r.h.s. of (186) owe their existence to the Lie - algebraic noncommutativity assumed in the present work. Most significantly

$$
\mathcal{R}_{3}+\mathcal{R}_{4}=0
$$


The vanishing of first order correction of the Ricci scalar for constant noncommutativity is well known [44. Here we find the same result for the Lie-algebraic structure. From the present analysis it is clear that various symmetries of the Riemann tensor and the spin connection of the commutative theory are responsible for the non existence of order $\theta$ correction. Thus it seems that the zero value of the first order correction is due to the underlying symmetries of space time which will presumably hold for more general noncommutative structure. However, we are unable to give a definitive proof of this.

\subsection{Noncommutativity Inspired Black Hole Physics}

We will now study some applications of NC gravity in Black Hole physics. We have seen that a direct generalisation of Einstein's theory of gravity to noncommutative spacetime results in $\mathrm{NC}$ correction which is a second order effect. However, this small correction incorporated in different phenomenology has been shown to produce important physical effects [45, 102, 103, 113. In these works the space-time of noncommutative theory is taken to be of Minkowski type, endowed with spherical noncommutative coordinates. A deformation of the gravitational field is constructed by gauging the noncommutative de Sitter $S O(4,1)$ group [94] and using SeibergWitten (SW) map [4. The deformed gravitational gauge potentials (tetrad fields) $\hat{e}_{\mu}^{a}(x, \theta)$ are obtained by contraction of the noncommutative gauge group $S O(4,1)$ to the Poincaré (inhomogeneous Lorentz) group ISO $(3,1)$. The fields are expanded in perturbative series where the different terms of the series are obtained from the commutative solution of the metric. The deformed gauge fields up to the second order in the noncommutativity parameters $\theta^{\mu \nu}$ are found. The correction terms require the commutative tetrad fields of the de Sitter gauge theory of gravitation over Minkowski spacetime. These solutions are found using a spherically symmetric ansatz and solving the corresponding Einstein equations. From the NC tetrad fields $\hat{e}_{\mu}^{a}(x, \theta)$ we construct the NC Reissner-Nordstrom metric $\hat{g}_{\mu \nu}(x, \theta)$. Naturally, the nontrivial correction starts from the second order. We explicitly calculate this leading $\mathrm{NC}$ correction term to the Reissner-Nordstrom metric. These solutions have been used to study the NC deformation of the charged black hole solutions [102, 103]. The deformed metric is calculated by the formula:

$$
\hat{g}_{\mu \nu}(x, \theta)=\frac{1}{2} \eta_{a b}\left(\hat{e}_{\mu}^{a} \star \hat{e}_{\nu}^{b+}+\hat{e}_{\nu}^{b} \star \hat{e}_{\mu}^{a+}\right) .
$$

Here + means complex conjugation. Note that Chamseddine's formalism involves extra complex degrees of freedom. These are necessary to close the gauge algebra in the noncommutative framework. One is then faced with the problem of the spurious degrees of freedom that remain after the commutative limit is taken. For a discussion of this point see [94, 110].

The constant antisymmetric matrix $\theta^{\mu \nu}$ can always be rotated to a skew-diagonal form 93 . We further assume vanishing noncommutativity in the time-space sector, which is quite usual in the literature. The non-zero components of the tetrad fields $\hat{e}_{\mu}^{a}(x, \theta)$ corresponding to this NC structure can be easily worked out using GRTensor II package of Maple. Then using the definition of the metric (188) we arrive at the following non-zero components of the deformed metric $\hat{g}_{\mu \nu}$ up to the second order in $\theta$. The explicit form of the non-zero components of the 
NC Reissner-Nordstrom metric computed in this way 102 are

$$
\begin{aligned}
\hat{g}_{00} & =-\left(1-\frac{2 M}{r}+\frac{Q^{2}}{r^{2}}\right)-\frac{1}{r^{6}}\left[M r^{3}-\frac{11 M^{2}+9 Q^{2}}{4} r^{2}-\frac{17 M Q^{2}}{4} r-\frac{7 Q^{4}}{2}\right] \theta^{2}+O\left(\theta^{4}\right) \\
\hat{g}_{11} & =\frac{1}{\left(1-\frac{2 M}{r}+\frac{Q^{2}}{r^{2}}\right)}+\frac{\left[-2 M r^{3}+3\left(M^{2}+Q^{2}\right) r^{2}-6 M Q^{2} r+2 Q^{4}\right]}{4 r^{2}\left(r^{2}-2 M r+Q^{2}\right)^{2}} \theta^{2}+O\left(\theta^{4}\right) \\
\hat{g}_{22} & =r^{2}+\frac{1}{16}\left[1-\frac{15 M}{r}+\frac{26 Q^{2}}{r^{2}}+\frac{4\left(M r-Q^{2}\right)^{2}}{r^{2}\left(r^{2}-2 M r+Q^{2}\right)}\right] \theta^{2}+O\left(\theta^{4}\right) \\
\hat{g}_{33} & =r^{2} \sin ^{2} \phi+\frac{1}{16}\left[\frac{4 r^{2}\left(M^{2}-M r\right)+8 Q^{2}\left(r^{2}-2 M r\right)+8 Q^{4}}{r^{2}\left(r^{2}-2 M r+Q^{2}\right)^{2}} \sin ^{2}+\cos ^{2} \phi\right] \theta^{2}+O\left(\theta^{4}\right)
\end{aligned}
$$

If we substitute $Q=0$ in our expressions (189) the solutions exactly reduces to the NC Schwarzschild solutions [45].

The NC corrections to the charged black hole solution may be obtained from the deformed metric. For instance we consider the event horizon. In commutative space-time we can identify the event horizons by following radial null curves and locating the radius at which $\frac{d t}{d r}$ becomes infinity. Following this and remembering that our event horizons should go to the commutative results in the limit $\theta \rightarrow 0$ we define the event horizons of the $\mathrm{NC} \mathrm{R}-\mathrm{N}$ metric from $g_{00}=0$. From our NC R-N solutions (189) it is straightforward to derive

$$
r^{2}-2 m r+Q^{2}=-\frac{\theta^{2}}{4 r^{4}}\left(4 m r^{3}-11 m^{2} r^{2}+9 Q^{2} r^{2}-17 m Q^{2} r+14 Q^{4}\right)
$$

the solutions to which give the horizon radii. Naturally we look for solutions correct up to second order in $\theta$. The required solutions are

$$
\begin{aligned}
& r_{+}=M+\sqrt{M^{2}-Q^{2}}+\frac{\theta^{2}}{2} \frac{A_{+}}{\sqrt{M^{2}-Q^{2}}} \\
& r_{-}=M-\sqrt{M^{2}-Q^{2}}-\frac{\theta^{2}}{2} \frac{A_{-}}{\sqrt{M^{2}-Q^{2}}}
\end{aligned}
$$


with $A_{+}$and $A_{-}$given by

$$
\begin{aligned}
A_{+}= & \frac{6 M^{4}+10 M^{3}\left(M^{2}-Q^{2}\right)^{(1 / 2)}+36 Q^{2} M^{2}-4 M\left(M^{2}-Q^{2}\right)^{(3 / 2)}}{4\left(M+\sqrt{M^{2}-Q^{2}}\right)^{4}} \\
& +\frac{35 Q^{2} M\left(M^{2}-Q^{2}\right)^{(1 / 2)}+5 Q^{4}}{4\left(M+\sqrt{M^{2}-Q^{2}}\right)^{4}} \\
A_{-}= & \frac{6 M^{4}-10 M^{3}\left(M^{2}-Q^{2}\right)^{(1 / 2)}+36 Q^{2} M^{2}+4 M\left(M^{2}-Q^{2}\right)^{(3 / 2)}}{4\left(M-\sqrt{M^{2}-Q^{2}}\right)^{4}} \\
& -\frac{35 Q^{2} M\left(M^{2}-Q^{2}\right)^{(1 / 2)}+5 Q^{4}}{4\left(M-\sqrt{M^{2}-Q^{2}}\right)^{4}}
\end{aligned}
$$

Note that these solutions properly map to the familiar (commutative) $\mathrm{R}-\mathrm{N}$ horizon

$$
r_{ \pm}=M \pm \sqrt{M^{2}-Q^{2}}
$$

in the limit $\theta \rightarrow 0$. As a result of the $\mathrm{NC}$ effect the distance between the event horizon radii increases. Also similar calculations have been performed for the Robertson Walker metric [113].

In the above we have discussed the deformation of general relativity in noncommutative space time. Our approach was perturbative (through the SW map 4]). Thus the essential nonlocality inherent in the NC field theories was not taken into account. Moreover in this approach it is not clear how to incorporate the basic symmetries in the model. As mentioned earlier an alternative approach was proposed where the effect of noncommutativity has been introduced in the level of the mean value [47, 111, 112. In the following section some thermodynamic applications of this approach is reviewed.

\subsection{Coherent State Based Approach to the Noncommutative Black Hole Physics}

The alternative approach towards incorporating $\mathrm{NC}$ effects is based on the coherent state formalism[46] of quantum optics. The idea is to modify the volume density to introduce the fuzziness corresponding to the NC structure. The method is exemplified in the following.

NC Schwarzschild black hole: A point particle of mass $M$ is described by the following volume density

$$
\rho=M \delta^{3}(\mathbf{r})
$$

but in a noncommutative space, concept of point does not exist. Instead, there is a smearing which comes as a consequence of position-position uncertainty relation. We introduce the 
noncommutative correction in (194) by replacing the Dirac delta function by a Gaussian distribution of minimal width $\sqrt{\theta}$

$$
\rho_{\theta}=\frac{M}{(4 \pi \theta)^{\frac{3}{2}}} e^{-\frac{r^{2}}{4 \theta}}
$$

where the noncommutative parameter $\theta$ is considered to be a small $\left(\sim\right.$ Planck length $\left.{ }^{2}\right)$ positive number. Using the above expression one can find the mass of a black hole by integrating (195) over a volume of radius $r$. This is found to be,

$$
m_{\theta}(r)=\int_{0}^{r} 4 \pi r^{\prime 2} \rho_{\theta}\left(r^{\prime}\right) d r^{\prime}=\frac{2 M}{\sqrt{\pi}} \gamma\left(\frac{3}{2}, \frac{r^{2}}{4 \theta}\right)
$$

where $\gamma\left(\frac{3}{2}, \frac{r^{2}}{4 \theta}\right)$ is the lower incomplete gamma function defined in the following way

$$
\gamma(a, x)=\int_{0}^{x} t^{a-1} e^{-t} d t
$$

In the $\theta \rightarrow 0$ limit it becomes the usual gamma function $\left(\Gamma_{\text {total }}(a)=\gamma(a, x)+\Gamma(a, x)\right)$.

$$
\Gamma_{\text {total }}=\int_{0}^{\infty} t^{a-1} e^{-t} d t
$$

and $m_{\theta}(r)$ of (196) reduces to $M$. Substituting (196) in the mass term of the Schwarzschild space time

$$
d s^{2}=-\left(1-\frac{2 M}{r}\right) d t^{2}+\left(1-\frac{2 M}{r}\right)^{-1} d r^{2}+r^{2} d \Omega^{2}
$$

we get the noncommutative Schwarzschild metric,

$$
d s^{2}=-\left(1-\frac{4 M}{r \sqrt{\pi}} \gamma\left(\frac{3}{2}, \frac{r^{2}}{4 \theta}\right)\right) d t^{2}+\left(1-\frac{4 M}{r \sqrt{\pi}} \gamma\left(\frac{3}{2}, \frac{r^{2}}{4 \theta}\right)\right)^{-1} d r^{2}+r^{2} d \Omega^{2}
$$

Above metric can also be interpreted as the solution of the Einstein equation $\left(G_{\theta}\right)^{\mu \nu}=8 \pi\left(T_{\theta}\right)^{\mu \nu}$. Here the $T_{\theta}$ is the energy momentum tensor

$$
\left(T_{\theta}\right)_{\mu}^{\nu}=\operatorname{diag}\left[-\rho_{\theta}, p_{r}, p^{\prime}, p^{\prime}\right]
$$

where, $p_{r}=-\rho_{\theta}$ and $p^{\prime}=p_{r}-\frac{r}{2} \partial_{r} \rho_{\theta}$ so that $\left(T_{\theta}\right)_{\mu ; \nu}^{\nu}=0$. The expression of $p^{\prime}$

$$
p^{\prime}=\left(\frac{r^{2}}{4 \theta}-1\right) \frac{M}{(4 \pi \theta)^{\frac{3}{2}}} \mathrm{e}^{-\frac{r^{2}}{4 \theta}}
$$

reduces to $p_{r}$ when $r \ll \sqrt{\theta}$. In the other limit $(r \gg \sqrt{\theta})$ the energy momentum tensor is zero and one recovers the Schwarzschild vacuum. Thus in the small length scale the radial

\footnotetext{
${ }^{5} \Gamma(a, x)=\int_{x}^{\infty} t^{a-1} e^{-t} d t$ is the upper incomplete gamma function
} 
pressure nullifies the gravitational attraction and hence collapse is prevented. Previously such phenomenon was associated with the presence of a de-Sitter metric inside the black hole 114 , 115. Here in the small $r$ limit

$$
-g_{t t}=1-\frac{M r^{2}}{3 \sqrt{\pi} \theta^{\frac{3}{2}}}
$$

For (203) the metric (200) becomes a de-Sitter metric with cosmological constant

$$
\Lambda=\frac{M}{3 \sqrt{\pi} \theta^{\frac{3}{2}}}
$$

having the following constant curvature

$$
R=\frac{4 M}{\sqrt{\pi} \theta^{\frac{3}{2}}}
$$

As a result we get a de-Sitter core of positive curvature surrounding the singularity.

Note that the metric (200) is stationary, static and spherically symmetric in nature - this fact has immense importance in the subsequent thermodynamic analysis. The event horizon $\left(r_{h}\right)$ for the metric (200) can be found by setting $g_{t t}\left(r_{h}\right)=0$. The result obtained is

$$
r_{h}=\frac{4 M}{\sqrt{\pi}} \gamma\left(\frac{3}{2}, \frac{r_{h}^{2}}{4 \theta}\right)
$$

which can not be solved in a closed form.

In Figure (11) we plot the black hole mass $M$ as a function of $r_{h}$ (for equation (206)). Clearly at $r_{h}=3.0 \sqrt{\theta}, M$ is minimum $\left(M_{\min }=1.9 \sqrt{\theta}\right)$ and when $M<M_{\min }$ no horizon is possible. This counter intuitive result has no analogy in the standard commutative space description of Schwarzschild black hole [47, 116, 117]. When $M>M_{\min }$ two different horizons appear one of them is outer (event) horizon and the other is inner (Cauchy) horizon. These two horizons merge as $M \rightarrow M_{\min }$. In the special case when $M$ is very large compared to $M_{\min }$ the inner horizon shrinks to zero and the outer horizon approaches the Schwarzschild radius 2M. Detailed discussions on these results may be found in [47, 116].

In the large radius regime $\left(\frac{r_{h}^{2}}{4 \theta}>>1\right)$ the asymptotic expansion of the lower incomplete $\gamma$ function

$$
\begin{aligned}
\gamma\left(\frac{3}{2}, x\right) & =\Gamma_{\text {total }}\left(\frac{3}{2}\right)-\Gamma\left(\frac{3}{2}, x\right) \\
& \simeq \frac{\sqrt{\pi}}{2}\left[1-e^{-x} \sum_{p=0}^{\infty} \frac{x^{\frac{1-2 p}{2}}}{\Gamma_{\text {total }}\left(\frac{3}{2}-p\right)}\right]
\end{aligned}
$$

is used to solve (206) by iteration. Keeping up to the order $\frac{1}{\sqrt{\theta}} e^{-\frac{M^{2}}{\theta}}$, we find

$$
r_{h} \simeq 2 M\left(1-\frac{2 M}{\sqrt{\pi \theta}} e^{-\frac{M^{2}}{\theta}}\right)
$$


Black hole temperature: The surface gravity $\left(\kappa\left(r_{h}\right)\right)$ for the classical noncommutative Schwarzschild spacetime (200) is given by

$$
\kappa\left(r_{h}\right)=\frac{1}{2}\left[\frac{d g_{00}}{d r}\right]_{r=r_{h}}=\frac{1}{2}\left[\frac{1}{r_{h}}-\frac{r_{h}^{2}}{4 \theta^{\frac{3}{2}}} \frac{e^{-\frac{r_{h}^{2}}{4 \theta}}}{\gamma\left(\frac{3}{2}, \frac{r_{h}^{2}}{4 \theta}\right)}\right] .
$$

The expression of surface gravity (209) is true when there is no back reaction. In order to find the appropriate modification in the presence of back reaction we first fix the units $G=c=k_{\mathrm{B}}=1$, in which 6 Planck length $l_{\mathrm{p}}=$ Planck mass $M_{\mathrm{p}}=\sqrt{\hbar}$. Since a loop expansion is equivalent to an expansion in powers of the Planck constant, the one loop back reaction effect in the surface gravity is written as,

$$
\mathcal{K}\left(r_{h}\right)=\kappa\left(r_{h}\right)+\xi \kappa\left(r_{h}\right)
$$

where $\xi$ is a dimensionless constant having magnitude of the order of $\hbar$. From dimensional arguments, therefore, it has the structure,

$$
\xi=\beta \frac{M_{\mathrm{p}}^{2}}{m_{\theta}^{2}}
$$

where $\beta$ is a pure numerical factor. In the commutative picture $\beta$ is known to be related to the trace anomaly coefficient [118, 119]. Putting this form of $\xi$ in (210) we get,

$$
\mathcal{K}=\kappa\left(r_{h}\right)\left(1+\beta \frac{M_{\mathrm{p}}^{2}}{m_{\theta}^{2}}\right)
$$

A similar expression was obtained earlier in [120, 118] for the commutative case. Eq. (212) is recast as,

$$
\mathcal{K}=\kappa\left(r_{h}\right)\left(1+\frac{\alpha}{m_{\theta}^{2}\left(r_{h}\right)}\right)
$$

where $\alpha=\beta M_{\mathrm{p}}^{2}$.

In order to write the above equation completely in terms of $r_{h}$ we have to express the mass $m_{\theta}$ in terms of $r_{h}$. For that we compare eqs. (196) and (206) to get,

$$
m_{\theta}\left(r_{h}\right)=\frac{r_{h}}{2}
$$

It is noteworthy that the structure of the above equation is identical to its commutative version.

Substituting (214) in (213) we get the value of modified noncommutative surface gravity

$$
\mathcal{K}=\frac{1}{2}\left[\frac{1}{r_{h}}-\frac{r_{h}^{2}}{4 \theta^{\frac{3}{2}}} \frac{e^{-\frac{r_{h}^{2}}{4 \theta}}}{\gamma\left(\frac{3}{2}, \frac{r_{h}^{2}}{4 \theta}\right)}\right]\left(1+\frac{4 \alpha}{r_{h}^{2}}\right)
$$

\footnotetext{
${ }^{6}$ Planck length $l_{P}=\left(\hbar G / c^{3}\right)^{1 / 2}$, Planck mass $M_{P}=(\hbar c / G)^{1 / 2}$
} 
So the noncommutative Hawking temperature including the effect of back reaction is given by,

$$
T_{h}=\frac{\mathcal{K}}{2 \pi}=\frac{1}{4 \pi}\left[\frac{1}{r_{h}}-\frac{r_{h}^{2}}{4 \theta^{\frac{3}{2}}} \frac{e^{-\frac{r_{h}^{2}}{4 \theta}}}{\gamma\left(\frac{3}{2}, \frac{r_{h}^{2}}{4 \theta}\right)}\right]\left(1+\frac{4 \alpha}{r_{h}^{2}}\right)
$$

If the back reaction is ignored (i. e. $\alpha=0$ ), the expression for the Hawking temperature is

$$
T_{h}=\frac{1}{4 \pi}\left[\frac{1}{r_{h}}-\frac{r_{h}^{2}}{4 \theta^{\frac{3}{2}}} \frac{e^{-\frac{r_{h}^{2}}{4 \theta}}}{\gamma\left(\frac{3}{2}, \frac{r_{h}^{2}}{4 \theta}\right)}\right]
$$

which agrees with the temperature obtained in [47]. Also for the commutative space limit $(\theta \rightarrow 0)$ we get

$$
T_{h}=\frac{1}{4 \pi r_{h}}\left(1+\frac{4 \alpha}{r_{h}^{2}}\right)
$$

Since in the limit $\theta \rightarrow 0, r_{h} \rightarrow 2 M$ above equation is written in the standard form

$$
T_{h}=T_{H}\left(1+\frac{\alpha}{M^{2}}\right)
$$

where $T_{H}=\frac{1}{8 \pi M}$ is the semiclassical temperature of the Schwarzschild black hole. This matches with the result previously found in [119, 121].

One problem with (219) is that as $M$ approaches zero due to Hawking radiation $T_{h}$ diverges. This is the Hawking paradox. We shall study the effect of noncommutativity and back reaction on this paradox by graphical methods when the black hole horizon is comparable to $\sqrt{\theta}$. The dependence of the temperature $T_{h}$ on the horizon radius $r_{h}$ is plotted in fig.(2) (with positive $\alpha$ ) and in fig.(3) (with negative $\alpha$ ).

Fig.(2) shows that the effect of back reaction does not change the qualitative nature of the graphs for both commutative and noncommutative cases. Though all curves marge at large $r_{h}$, in the region $r_{h} \simeq \sqrt{\theta}$ the noncommutative effect is quite significant. The commutative curves diverge as $r_{h} \rightarrow 0$ whereas the noncommutative curves (with or without back reaction) are no longer of hyperbolic type, instead they have a peak at $r_{h} \simeq 4.7 \sqrt{\theta}$ and then fall quickly to zero at $r_{0}=3 \sqrt{\theta}$. This $r_{0}$ corresponds to the radius of the extremal black hole. In the region $r_{h}<r_{0}$ the temperature is negative which means there is no black hole. In this way Hawking paradox can be avoided by means of introducing noncommutativity. This point was first noted in [47].

In fig.(3) we see that, for $\alpha \neq 0, \theta=0$ (red curve) $T_{h}$ becomes zero at $r_{h}=r_{0} \simeq 2.0 \sqrt{\theta}$. This together with the case $\alpha=0, \theta \neq 0$ (yellow curve of (2) and (3)) therefore avoid the Hawking paradox. But when $\alpha$ and $\theta$ are both nonzero $T_{h}$ vanishes for $r_{h} \simeq 3.0 \sqrt{\theta}$ and $r_{h}=2.0 \sqrt{\theta}$ and then it diverges. Since this is not meaningful physically, $\alpha$ must be positive for the cases where both noncommutativity and back reaction effects are present.

Entropy and the area law: Having obtained the Hawking temperature of the black hole we calculate the Bekenstein-Hawking entropy. The expression of entropy can be obtained from 
the second law of thermodynamics. Using (208) the temperature (216) can be approximately expressed in terms of $M$. To the leading order, we obtain,

$$
T_{h}=\frac{M^{2}+\alpha}{8 \pi M^{3}}\left[1-\frac{4 M^{5}}{\left(M^{2}+\alpha\right) \theta \sqrt{\pi \theta}} e^{-\frac{M^{2}}{\theta}}\right]+\mathcal{O}\left(\frac{1}{\sqrt{\theta}} e^{-\frac{M^{2}}{\theta}}\right)
$$

Using the second law of thermodynamics $d S_{\mathrm{bh}}=\frac{d M}{T_{h}}$ the Bekenstein-Hawking entropy is found to be

$$
\begin{aligned}
S_{\mathrm{bh}} & \simeq 4 \pi M^{2}-4 \pi \alpha \ln \left(\frac{M^{2}}{\alpha}+1\right) \\
& \left.-16 \sqrt{\frac{\pi}{\theta}} M^{3} e^{-\frac{M^{2}}{\theta}}+\text { const.(independent of } M\right)
\end{aligned}
$$

In order to see the connection between entropy and the horizon area we need to write the right hand side of above equation in terms of the area. But the effect of back reaction on a metric is unknown. So we take $\alpha \rightarrow 0$ limit in the above equation to get

$$
S_{\mathrm{bh}}=\int \frac{d M}{T_{h}} \simeq 4 \pi M^{2}-16 M^{3} \sqrt{\frac{\pi}{\theta}} e^{-\frac{M^{2}}{\theta}} .
$$

The same expression of Bekenstein-Hawking entropy can also be obtained by the tunnelling method as shown earlier in [117]. Now using (208) we obtain the noncommutative horizon area $(A)$,

$$
A=4 \pi r_{h}^{2}=16 \pi M^{2}-64 \sqrt{\frac{\pi}{\theta}} M^{3} e^{-\frac{M^{2}}{\theta}} .
$$

Comparing equations (222) and (223) we find that at the leading order, the noncommutative black hole entropy satisfies the area law,

$$
S_{\mathrm{bh}}=\frac{A}{4}
$$

which is functionally identical to the Benkenstein-Hawking area law in the commutative space. This naturally arises the question whether this law is true up to all orders in $\theta$ or not. To address this issue we follow a method based on graphical analysis. First we write the second law of thermodynamics in terms of the horizon $r_{h}$ of the black hole. Using (206) and (217) we get close form relation

$$
\frac{d S_{\mathrm{bh}}}{d r_{h}}=\frac{\pi^{\frac{3}{2}} r_{h}}{\gamma\left(\frac{3}{2}, \frac{r_{h}^{2}}{4 \theta}\right)}
$$

On the other hand (223) and (224) yield,

$$
\left.\frac{d S_{\mathrm{bh}}}{d r_{h}}\right|_{\text {semiclassical }}=\frac{d S_{\mathrm{bh}}}{d A} \frac{d A}{d r_{h}}=2 \pi r_{h} .
$$


Though in the large $r_{h}$ limit (225) reduces to (226) when $r_{h} \simeq \sqrt{\theta}$ there is a mismatch. In fact we need a entropy-area law which is correct in the region $r_{h} \geq 3.0 \sqrt{\theta}$, because when $r_{h}$ is smaller than that black hole does not exist.

To find the corrections to the semiclassical area law we rewrite (225) in the following way

$$
\begin{aligned}
\frac{d S_{\mathrm{bh}}}{d r_{h}} & =\frac{\pi^{\frac{3}{2}} r_{h}}{\frac{\sqrt{\pi}}{2}-\Gamma\left(\frac{3}{2}, \frac{r_{h}^{2}}{4 \theta}\right)} \\
& =2 \pi r_{h}\left[1+\frac{2}{\sqrt{\pi}} \Gamma\left(\frac{3}{2}, \frac{r_{h}^{2}}{4 \theta}\right)+\frac{4}{\pi} \Gamma^{2}\left(\frac{3}{2}, \frac{r_{h}^{2}}{4 \theta}\right)+\ldots \ldots . .\right] .
\end{aligned}
$$

The claim is that the terms involving the upper incomplete gamma functions are the corrections to the area law. The justification is given by the help of graphical analysis. The graph of the above equation up to $\Gamma^{2}$ term is given in figure (44) along with equations (225) and (226). It shows that the blue curve coincides with the red curve for the entire physical domain $r_{h} \geq$ $3.0 \sqrt{\theta}$. Inclusion of the third order correction improves the situation even better[107]. Thus we integrate (227) over $r_{h}$ to obtain

$$
\begin{aligned}
S_{\mathrm{bh}} & =\pi r_{h}^{2}-\sqrt{\frac{\pi}{\theta}} r_{h}^{3} e^{-\frac{r_{h}^{2}}{4 \theta}}-\sqrt{\pi \theta} r_{h} e^{-\frac{r_{h}^{2}}{4 \theta}}-6 \pi \theta\left(1-\operatorname{Erf}\left(\frac{r_{h}}{2 \sqrt{\theta}}\right)\right) \\
& +2 \sqrt{\pi} r_{h}^{2} \Gamma\left(\frac{3}{2}, \frac{r_{h}^{2}}{4 \theta}\right)+8 \int r_{h} \Gamma^{2}\left(\frac{3}{2}, \frac{r_{h}^{2}}{4 \theta}\right) d r_{h} .
\end{aligned}
$$

Making use of (223), the above equation is finally written in terms of the noncommutative area as,

$$
\begin{aligned}
S_{\mathrm{bh}} & =\frac{A}{4}-\frac{A^{\frac{3}{2}}}{8 \pi \sqrt{\theta}} e^{-\frac{A}{16 \pi \theta}}-\frac{\sqrt{\theta A}}{2} e^{-\frac{A}{16 \pi \theta}}-6 \pi \theta\left(1-\operatorname{Erf}\left(\frac{1}{4} \sqrt{\frac{A}{\pi \theta}}\right)\right) \\
& +\frac{A}{2 \sqrt{\pi}} \Gamma\left(\frac{3}{2}, \frac{A}{16 \pi \theta}\right)+\frac{1}{\pi} \int \Gamma^{2}\left(\frac{3}{2}, \frac{A}{16 \pi \theta}\right) d A .
\end{aligned}
$$

The first term is the noncommutative version of the Bekenstein-Hawking area law whereas the other terms are the different order corrections which contain exponentials of the noncommutative semiclassical area $A$ and the error function. Expectedly, in the limit $\theta \rightarrow 0$, all correction terms vanish and one recovers the celebrated Bekenstein-Hawking area law.

\section{Concluding Remarks:}

In the present article we have tried to convey the flavors of noncommutative geometry inspired physics that have created a lot of interest in recent times. Indeed the study is by no means exhaustive simply because the topic is too vast and varied to be covered meaningfully in a single article. Nevertheless this study is representative, covering those aspects which have both physical and mathematical interest. 
From the physical perspective the effect of change of statistics in many body systems presents an intriguing situation. Likewise, as this review shows, Doubly Special Relativity acts as a link between noncommutativity and quantum gravity. Noncommutativity also prevents the singularity problem of Schwarzschild black hole by preventing its absolute collapse. The presence of noncommutativity naturally acts like a de-Sitter core beyond which no further collapse takes place.

From the mathematical point of view a detailed study of twisted gauge symmetries was given. This is normally left out in standard articles on twists which concentrate basically on twisted Poincare symmetry. The present discussion therefore compliments existing analysis. Moreover it was reassuring to observe that approaches used in conventional gauge theories with appropriate modifications were revealed even in the noncommutative context. Although the specific topics we have covered are far from being completely understood and we hope that this article will provide the necessary impetus for further research.

\section{Acknowledgments}

We thank Shailesh Kulkarni and Sudipta Das for their help. 


\section{References}

[1] M.R.Douglas and N.A.Nekrasov, Rev. Mod. Phys. 73977 (2001) hep-th/0106048]; R. J. Szabo, Phys. Rep. 378207 (2003) hep-th/0109162].

[2] H. S. Snyder, Phys. Rev. 7138 (1947); ibid 7268 (1947).

[3] C. N. Yang, Phys. Rev. 72874 (1947) .

[4] N. Seiberg, E. Witten, JHEP 9909032 (1999) hep-th/9908142.

[5] M. M. Sheikh-Jabbari, Phys. Lett. B 455, 129 (1999) hep-th/9901080|; V. Schomerus, JHEP 06030 (1999) hep-th/9903205]; P. M. Ho and Y.-S. Wu, Phys. Lett. B 39852 (1997) [hep-th/9611233]; ibid Phys. Rev. D 58, 066003 (1998) [hep-th/9801147].

[6] R. Banerjee, B. Chakraborty and S.Ghosh, Phys. Lett. B 537340 (2002) [hep-th/0203199]; R. Banerjee, B. Chakraborty and K. Kumar, Nucl. Phys. B 668179 (2003) (hep-th/0306122); B. Chakraborty, S. Gangopadhyay, A. G. Hazra and F. G. Scholtz, Phys.Lett. B 625302 (2005) [hep-th/0508156]; C. Chatterjee, S. Gangopadhyay, A. Ghosh Hazra, S. Samanta Int. J. Theor. Phys. 472372 (2008) arXiv:0801.4189.

[7] B. Chakraborty, S. Gangopadhyay and A. G. Hazra, Phys. Rev. D 74105011 (2006) [hep-th/0608065].

[8] R. Gopakumar, S. Minwalla and A. Strominger, JHEP 0005020 (2000) hep-th/0003160]; K. Dasgupta, S. Mukhi and G. Rajesh, JHEP 0006022 (2000) hep-th/0005006; J. A. Harvey, P. Kraus, F. Larsen and E. J. Martinec, JHEP 0007042 (2000) [hep-th/0005031]; M. Hamanaka, hep-th/0303256.

[9] V. P. Nair and A. P. Polychronakos, Phys. Rev. Lett. 87030403 (2001) [hep-th/0102181]; J. A. Harvey, hep-th/0105242.

[10] R. Banerjee, H. Seok Yang Nucl. Phys. B 708434 (2005) |hep-th/0404064|; V. O. Rivelles Phys. Lett. B 558191 (2003) [hep-th/0212262].

[11] V. O. Rivelles, hep-th/0305122 ; E. Harikumar, V. O. Rivelles Class. Quant. Grav. 237551 (2006) hep-th/0607115; P. Aschieri, M. Dimitrijevic, F. Meyer, J. Wess, Class. Quant. Grav. 231883 (2006) hep-th/0510059]; P. Aschieri, C. Blohmann, M. Dimitrijevic, F. Meyer, P. Schupp, J. Wess, Class. Quant. Grav. 223511 (2005) [hep-th/0504183].

[12] J. M. Gracia-Bondia and C. P. Martin, Phys. Lett. B 479321 (2000) hep-th/0002171; L. Bonora, M. Schnabl and A. Tomasiello, Phys. Lett. B 485311 (2000) |hep-th/0002210]; F. Ardalan and N. Sadooghi, Int. J. Mod. Phys. A 163151 (2001) hep-th/0002143]; C. P. Martin, Nucl. Phys. B 623150 (2002) hep-th/0110046]; R. Banerjee and S. Ghosh Phys. Lett. B 533162 (2002) [hep-th/0110177]; R. Banerjee, K. Kumar Phys. Rev. D 75 045008 (2007) hep-th/0604162 ]; ibid, Phys. Rev. D 72085012 (2005) [hep-th/0505245]; ibid, Phys. Rev. D 71045013 (2005) [hep-th/0404110|; R. Banerjee, C. Lee, H. S. Yang Phys. Rev. D 70065015 (2004) arXiv:hep-th/0312103]; R. Banerjee Int. J. Mod. Phys. A 19613 (2004) hep-th/0301174. 
[13] S. Ghosh, Nucl. Phys. B 670359 (2003) [hep-th/0306045]; ibid Phys. Rev. D 70085007 (2004) [hep-th/0402029]; ibid Annals. Phys. 318432 (2005); T. R. Govindarajan, E. Harikumar, Phys.Lett. B 602238 (2004) [hep-th/0406273]; B. Chakraborty , S. Ghosh and R. P. Malik, Nucl. Phys. B 600351 (2001) |hep-th/0008168|.

[14] S. Ghosh, Phys. Lett. B 563112 (2003) |hep-th/0303022];ibid Phys. Lett. B 558245 (2003) [hep-th/0210107], Mod. Phys. Lett. A 201227 (2005) hep-th/0407086]; M. Botta Cantcheff and P.Minces, Phys. Lett. B 557283 (2003) (hep-th/0212031); O. F. Dayi, Phys. Lett. B 560239 (2003) [hep-th/0302074]; M. Botta Cantcheff and P. Minces, Eur. Phys. J. C 34393 (2004) [hep-th/0306206]; M. S. Guimaraes, D. C.Rodrigues, C. Wotzasek and J. L. Noronha, Phys. Lett. B 605419 (2005) |hep-th/0410156|; E. Harikumar, Victor O. Rivelles Phys. Lett. B 625156 (2005) [hep-th/0506078]; ibid hep-th/0610098.

[15] R. Banerjee, S. Ghosh, T. Shreecharan, Phys. Lett. B 662231 (2008) arXiv:0712.3631.

[16] C. P. Martin, C. Tamarit, Phys. Lett. B 658170 (2008) [arXiv:0706.4052].

[17] V. P. Nair and A. P. Polychronakos, Phys. Lett. B 505267 (2001) hep-th/0011172; J. Gamboa, M. Loewe, and J. C. Rojas, Phys. Rev. D 64067901 (2001) hep-th/0010220|; S. Bellucci, A. Nersessian, and C. Sochichiu, Phys. Lett. B 522345 (2001) |hep-th/0106138]; R. Banerjee, Mod. Phys. Lett. A 17631 (2002) (hep-th/0106280) ; B. Muthukumar and P. Mitra, Phys. Rev. D 66027701 (2002) |hep-th/0204149]; S. Samanta Mod. Phys. Lett. A 21675 (2006) hep-th/0510138] ; S. Gangopadhyay, F. G. Scholtz, arXiv:0812.3474.

[18] M. Chaichian, M. M. Sheikh-Jabbari and A. Tureanu, Phys. Rev. Lett. 86, 2716 (2001) arXiv:hep-th/0010175]; X. Calmet, Eur.Phys.J.C41:269 (2005) arXiv:hep-ph/0401097]; Z. Guralnik, R. Jackiw, S.-Y. Pi and A. P. Polychronakos, Phys. Lett. B517, 450 (2001) arXiv:hep-th/0106044].

[19] O. Bertolami, J. G. Rosa, C. M. L. de Aragao, P.Castorina and D. Zappala, Phys. Rev. D 72, 025010 (2005) (hep-th/0505064); R. Banerjee, B. Dutta Roy, S. Samanta Phys.Rev.D74:045015,2006 (hep-th/0605277); A. Saha, arXiv:0803.3957; S. Samanta, arXiv:0804.0172.

[20] T. Filk, Phys. Lett. B 37653 (1996); N. Ishibashi, S. Iso, H. Kawai and Y. Kitazawa, Nucl. Phys. B 573573 (2000) [hep-th/9910004]; S. Minwalla, M. Van Raamsdonk and N. Seiberg, JHEP 0002020 (2000) hep-th/9910004].

[21] H. Grosse and R. Wulkenhaar, Commun. Math. Phys. 256305 (2005) hep-th/0305066); E. Langmann, R. J. Szabo and K. Zarembo, JHEP 0401017 (2004) hep-th/0308043]; H. Grosse and R. Wulkenhaar, Eur. Phys. J. C 35277282 (2004) hep-th/0402093; V. Rivasseau, Seminaire Poincare X, 2007.

[22] A. Connes and M. A. Rieffel, Contemp. Math. 62237 (1987) ; A. Connes and J. Lott, Nucl. Phys. (Proc. Suppl.) B 1829 (1990); C.P. Martin, J. M. Gracia-Bond and J. C. V rilly, Phys. Rep. 294363 (1998) [hep-th/9605001]; Ali H. Chamseddine, A. Connes Phys. Rev. Lett. 99191601 (2007) arXiv:0706.3690]; A. Connes, JHEP 0611081 (2006) arXiv:hep-th/0608226]; A. Connes, Seminaire Poincare X, 2007.

[23] L. Susskind, arXiv: hep-th/0101029; A. P. Polychronakos, JHEP 0104011 (2001) [hep-th/0103013]; A. P. Polychronakos, Seminaire Poincare X, 2007. 
[24] C. Duval, P. A. Horvathy, Phys. Lett. B 594402 (2004) arXiv:hep-th/0402191]; V. P. Nair and A. P. Polychronakos, Phys. Lett. B 505267 (2001) |hep-th/0011172|.

[25] M. S. Plyushchay, Phys. Lett. B 248107 (1990); P. A. Horvathy, M. S. Plyushchay, Phys. Lett. B 595547 (2004); P. A. Horvathy, M. S. Plyushchay, Nucl. Phys. B 714269 (2005); R. Jackiw and V. P. Nair, Phys. Rev D 431933 (1991); C. Chou, V. P. Nair and A. P. Polychronakos, Phys. Lett. B 304105 (1993) hep-th/9301037|; S. Ghosh, Phys. Lett. B 338235 (1994), Erratum-ibid. B 347 468(1995) |hep-th/9406089]; Gerald V. Dunne, R. Jackiw, C. A. Trugenberger, Phys. Rev. D 41661 (1990).

[26] F. G. Scholtz, B. Chakraborty, S. Gangopadhyay, J. Govaerts J. Phys. A 389849 (2005) cond-mat/0509331.

[27] A. Berard and H. Mohrbach, Phys. Rev. D 69127701 (2004) hep-th/0310167; P. Gosselin, A. Brard, H. Mohrbach, Eur. Phys. J. B 58137 (2007) hep-th/0603192; K. Yu. Bliokh and Yu. P. Bliokh, Annals. Phys. 31913 (2005) quant-ph/0404144].

[28] T. Jungwirth, Q. Niu and A. H. M acdonald, Phys. Rev. Lett. 88207208 (2002); M. Onoda and N. Nagaosa, Phys. Rev. Lett. 93083901 (2004); D. Xiao, J. Shi and Q. Niu, Phys. Rev. Lett. 95137204 (2005) cond-mat/0502340; C. Duval, Z. Horvath, P. A. Horvathy and L. Martina, Phys. Rev. Lett. 96099701 (2006) cond-mat/0509806].

[29] S. Murakami, N. Nagaosa and S. C. Zhang, Science 3011348 (2003); J. Sinova, D. Culcer, Q. Niu, N. A. Sinitsyn, T. Jungwirth and A. H. Macdonald., Phys. Rev. Lett. 92126603 (2004); S. Dhar, B. Basu and S. Ghosh, Phys. Lett. A 371406 (2007) [cond-mat/0701096]

[30] P. Gosselin, A. Brard, H. Mohrbach and S. Ghosh, Eur. Phys. J. C 59883 (2009) arXiv:0802.3565.

[31] R. Jackiw, Phys. Rev. Lett. 54159 (1985); ibid Int. J. Mod. Phys. A19S1 137 (2004) hep-th/0212058.

[32] M. Chaichian, S. Ghosh, M. Langvik and A. Tureanu, Phys. Rev. D 79125029 (2009) arXiv:0902.2453.

[33] B. Chakraborty, S. Gangopadhyay, A. G. Hazra and F. G. Scholtz, J. Phys. A 399557 (2006) |hep-th/0601121|.

[34] E. Akofor, A. P. Balachandran, A. Joseph, Int. J. Mod. Phys. A 231637 (2008) arXiv:0803.4351.

[35] A. P. Balachandran, G. Mangano, A. Pinzul and S. Vaidya, Int. J. Mod. Phys. A 213111 (2006) hep-th/0508002.

[36] S. Ghosh and P. Pal, Phys. Rev. D 75105021 (2007) hep-th/0702159.

[37] G. Amelino-Camelia, Nature 41834 (2002) [gr-qc/0207049]; ibid Phys. Lett. B 510255 (2001) hep-th/0012238]; ibid Int. J. Mod. Phys. D11 35 (2002) [gr-qc/0012051].

[38] R. Banerjee and S. Samanta, JHEP 0702046 (2007) hep-th/0611249].

[39] R. Banerjee and S. Samanta Eur. Phys. J. C 51207 (2007) [arXiv: hep-th/0608214]. 
[40] J. Wess, J. Phys. Conf. Ser. 53752 (2006) [hep-th/0608135]; ibid hep-th/0408080.

[41] M. Chaichian and A. Tureanu, Phys. Lett. B 63799 (2006) hep-th/0604025];

[42] M. Chaichian, P. Prenajder, A. Tureanu, Phys. Rev. Lett. 94151602 (2005) [hep-th/0409096]; M. Chaichian, P. Kulish, K. Nishijima, A. Tureanu, Phys. Lett. B 604 98 (2004) hep-th/0408069].

[43] R. Banerjee, B. Chakraborty, K. Kumar Phys. Rev. D 70125004 (2004) [hep-th/0408197];

S. Ghosh, hep-th/0310155.

[44] P. Mukherjee and A. Saha, Phys. Rev. D 74027702 (2006) [hep-th/0605287].

[45] M. Chaichian, A. Tureanu and G. Zet, Phys. Lett B 660573 (2008) arXiv:0710.2075].

[46] R. J. Glauber, Phys. Rev. 1312766 (1963)

[47] P. Nicolini, A. Smailagic and E. Spallucci, Phys. Lett. B 547632 (2006) [gr-qc/0510112].

[48] V. G. Kupriyanov, D. V. Vassilevich, Eur. Phys. J. C 58627 (2008).

[49] N. Macris and S. Ouvry, J. Phys. A 354477 (2002) hep-th/0112181.

[50] F. G. Scholtz, B. Chakraborty, S. Gangopadhyay, A. Ghosh Hazra, Phys. Rev. D 71 085005 (2005) [hep-th/0502143].

[51] M. Chaichian, P. Kulish, K. Nishijima and A.Tureanu, Phys. Lett. B 60498 (2004) [hep-th/0408069].

[52] M. Dimitrijevic, L. Jonke, L. Moller, E. Tsouchnika, J. wess, Eur. Phys. J. C 31129 (2003) hep-th/0307149.

[53] S. Khan, B. Chakraborty and F. G. Scholtz, Phys. Rev. D 78025024 (2008) arXiv:0707.4410.

[54] P. G. Castro, B. Chakraborty and F. Toppan, J. Math. Phys. 49082106 (2008) arXiv:0804.2936.

[55] G. Fiore and J. Wess, Phys. Rev. D 75105022 (2007).

[56] G. Piacitelli, arXiv:0902.0575.

[57] A. P. Balachandran, T. R. Govindarajan, G. Mangano, A. Pinzul, B. Qureshi, S. Vaidya, Phys. Rev. D 75045009 (2007) [hep-th/0608179]; A. Tureanu, Phys. Lett. B 638296 (2006) [hep-th/0603219].

[58] F. G. Scholtz, B. Chakraborty, J. Govaerts and S.Vaidya, J. Phys. A 1458 (2007) arXiv:0709.3357.

[59] S. Gangopadhyay and F. G. Scholtz, Phys. Rev. Lett. 102241602 (2009) [arXiv:0904.0379].

[60] C. Duval, P. A. Horvathy, Phys. Lett. B 479284 (2000). 
[61] V. P. Nair, A. P. Polychronakos, Phys. Lett. B 505267 (2001).

[62] F. G. Scholtz, L. Gouba, A. Hafver, C. Rohwer, J. Phys. A 42175303 (2009) arXiv:0812.2803.

[63] F. G. Scholtz, J. Govaerts, J. Phys. A 41505003 (2008) [arXiv:0810.3064].

[64] C. Rovelli and L. Smolin, Nucl. Phys. B 442593 (1995); Erratum: ibid 456734 (1995) [C. Rovelli and L. Smolin].

[65] R. Gambini and J. Pullin, Phys. Rev. D 59124021 (1999) gr-qc/9809038]; T. Kifune,; Astrophys. J. Lett. 518, L21 (1999) astro-ph/9904164|; J. Alfaro, H.A. Morales-Tecotl and L.F. Urrutia, Phys. Rev. Lett. 842318 (2000) |gr-qc/9909079]; S. D. Biller et al., Phys. Rev. Lett. 832108 (1999) gr-qc/9810044]; J.P. Norris, J.T. Bonnell, G. F. Marani and J. D. Scargle, astro-ph/9912136; A. de Angelis, astro-ph/0009271; R. Aloisio, P. Blasi, P.L. Ghia and A.F. Grillo, Phys. Rev. D 62053010 (2000) |astro-ph/0001258]; R.J. Protheroe and H. Meyer, Phys. Lett. B 4931 (2000) astro-ph/0005349]. H. Sato, astro-ph/0005218.

[66] J. Magueijo and L. Smolin, Phys. Rev. Lett. 88190403 (2002) hep-th/0112090]; ibid Phys.Rev. D 67044017 (2003) gr-qc/0207085].

[67] J. Lukierski, A. Nowicki, H. Ruegg and V. N. Tolstoy, Phys. Lett. B264 331 (1991); S. Majid and H. Ruegg, Phys. Lett. B 334348 (1994) [hep-th/9405107|; J. Lukierski, H. Ruegg, W. J. Zakrzewski, Annals. Phys. 24390 (1995) [hep-th/9312153].

[68] S. Doplicher, K. Fredenhagen and J. E. Roberts, Phys. Lett. B 33139 (1994).

[69] J. Kowalski-Glikman, Lect. Notes Phys. 669131 (2005) hep-th/0405273.

[70] J. Lukierski, A. Nowicki and H. Ruegg, Phys. Lett. B 293344 (1992); J. Lukierski, H. Ruegg and W. Ruhl, Phys. Lett. B 313357 (1993).

[71] J. Kowalski-Glikman and S. Nowak, Phys. Lett. B 539126 (2002) [hep-th/0203040]; ibid Int. J. Mod. Phys. D12 299 (2003) [hep-th/0204245]; ibid Class. Quant. Grav. 204799 (2003) hep-th/0304101]; J. M. Romero and A. Zamora, Phys. Rev. D 70105006 (2004) hep-th/0408193.

[72] A. Granik, hep-th/0207113; S. Mignemi, Phys. Rev. D 68065029 (2003) gr-qc/0304029.

[73] D. Kimberly, J. Magueijo and J. Medeiros, Phys. Rev. D 70084007 (2004) [gr-qc/0303067].

[74] N. R. Bruno, G.A melino-Camelia and J.Kowalski-Glikman, Phys. Lett. B 522133 (2001) hep-th/0107039.

[75] F. Girelli, S. Liberati and L. Sindoni, Phys. Rev. D 75064015 (2007) gr-qc/0611024;; S. Hossenfelder, Phys. Lett. A 36711 (2007) hep-th/0612167.

[76] J. Antonio Garcia, Phys. Rev. D 76048501 (2007) arXiv:0705.0143; B. F. Rizzuti, arXiv:0710.3724.

[77] S. Meljanac and M. Stojic, Eur. Phys. J. C 47531 (2006) [hep-th/0605133]; S. Meljanac, S. Kresic-Juric and M. Stojic, Eur. Phys. J. C 51229 (2007) [hep-th/0702215]. 
[78] P. Gosselin, A. Berard, H. Mohrbach and S. Ghosh, Phys. Lett. B 660267 (2008) arXiv:0709.0579.

[79] A. Agostini, G. Amelino-Camelia and M. Arzano, Class. Quant. Grav. 212179 (2004) gr-qc/0207003|.

[80] A. A. Deriglazov, JHEP 0303021 (2003) hep-th/0211105]; A. Pinzul, A. Stern, Phys. Lett. B 593279 (2004) [hep-th/0402220]; S. Ghosh, Phys. Lett. B 648262 (2007) [hep-th/0602009]; ibid Phys. Rev. D 74084019 (2006) hep-th/0608206]; ibid Phys. Lett. B 623251 (2005) [hep-th/0506084]; S. Ghosh and P. Pal, Phys. Lett. B 618243 (2005) hep-th/0502192]; F. Girelli, T. Konopka, J. Kowalski-Glikman and E. R. Livine, Phys. Rev. D 73045009 (2006) [hep-th/0512107]; L. Freidel, F. Girelli and E. R. Livine, Phys. Rev. D 75105016 (2007) hep-th/0701113]; R. Banerjee, S. Kulkarni and S. Samanta, JHEP 0605077 (2006) [hep-th/0602151].

[81] P. A. M.Dirac, Lectures on Quantum Mechanics, Yeshiva University Press, New York, 1964.

[82] D. V. Vassilevich, Mod. Phys. Lett. A 211279 (2006) arXiv:hep-th/0602185

[83] P. Aschieri, M. Dimitrijevic, F. Meyer, S. Schraml and J. Wess Lett. Math. Phys. 7861 (2006) arXiv:hep-th/0603024.

[84] D. M. Gitman and I. V. Tyutin, Quantization of Fields with Constraints, Springer-Verlag Berlin, Heidelberg (1990).

[85] A. Shirzad, J. Phys. A 312747 (1998).

[86] R. Banerjee, H. J. Rothe and K. D. Rothe, Phys. Lett. B 479429 (2000) [hep-th/9907217]; arXiv:hep-th/9909039 R. Banerjee, H.J. Rothe, K.D. Rothe J.Phys. A33 2059 (2000).

[87] R. Banerjee, H. J. Rothe and K. D. Rothe, Phys. Lett. B 463248 (1999) [hep-th/9906072].

[88] M. Hennaux and C. Teitelboim, Quantization of Gauge Systems, Princeton University Press, Princeton (1992).

[89] L. Alvarez-Gaume, F. Meyer, M. A. Vazquez-Mozo, Nucl. Phys. B 75392 (2006) [hep-th/0605113].

[90] S. Samanta, arXiv:0708.3300.

[91] R. Amorim and F. A. Farias, Phys. Rev. D 65065009 (2002) hep-th/0109146].

[92] R. Banerje, Phys. Rev. D 67105002 (2003) hep-th/0210259.

[93] R. J. Szabo, Classical Quantum Gravity 23 R199 (2006) hep-th/0606233]; E. Langmann, R. J. Szabo, Phys. Rev. D 64104019 (2001) [hep-th/0105094].

[94] A. H. Chamseddine, Phys. Lett. B 50433 (2001) hep-th/0009153].

[95] P. Aschieri, C. Blohmann, M. Dimitrijevic, F. Meyer, P. Schupp, J. Wess Class. Quant. Grav. 223511 (2005) hep-th/0504183. 
[96] X. Calmet, A. Kobakhidze, Phys. Rev. D 72045010 (2005) hep-th/0506157.

[97] J. J. van der Bij, H. van Dam and Y. J. Ng, Physica 116A, 307 (1982); F. Wilczek, Phys. Rept. 104, 143 (1984); W. Buchmuller and N. Dragon, Phys. Lett. B 207292 (1988); M. Henneaux and C. Teitelboim, Phys. Lett. B 222, 195 (1989); W. G. Unruh, Phys. Rev. D 401048 (1989).

[98] X. Calmet, A. Kobakhidze, Phys. Rev. D 74047702 (2006) hep-th/0605275.

[99] R Banerjee, P. Mukherjee, S. Samanta, Phys. Rev. D 75125020 (2007) hep-th/0703128].

[100] E. Harikumar and Vivtor. O. Rivelles, Class. Quant. Grav. 237551 (2006) hep-th/0607115.

[101] A. Kobakhidze, arXiv:0712.0642 [gr-qc].

[102] P. Mukherjee and A. Saha, Phys. Rev. D 77064014 (2008) arXiv:0710.5847.

[103] M. Chaichian, M. R. Setare, A. Tureanu and G. Zet, JHEP 0804064 (2008) arXiv:0711.4546].

[104] M. Salizzoni, A. Torrielli, H. S. Yang, Phys. Lett. B 634427 (2006) hep-th/0510249]; H. S. Yang, M. Salizzoni, Phys. Rev. Lett. 96201602 (2006) hep-th/0512215]; H. S. Yang, hep-th/0608013; ibid, hep-th/0611174; ibid, Mod. Phys. Lett. A 221119 (2007) hep-th/0612231]; H. Steinacker, Nucl. Phys. B 8101 (2009) [arXiv:0806.2032]; D. Klammer, H. Steinacker, Phys. Rev. Lett. 102 221301(2009) arXiv:0903.0986].

[105] P. Nicolini, Int. J. Mod. Phys. A 241229 (2009) arXiv:0807.1939.

[106] E. D. Grezia, G. Esposito and G.Miele, Class. Quant. Grav. 236425 (2006) hep-th/0607157]; K.Nozari and S.H.Mehdipour, Class. Quant. Grav. 25175015 (2008) arXiv:0801.4074].

[107] R. Banerjee, B. Ranjan Majhi, S. K. Modak, Class. Quant. Grav. 26085010 (2009) arXiv:0802.2176].

[108] J. Madore, S. Schraml, P. Schupp, J. Wess Eur. Phys. J. C18 78 (2001) [hep-th/0009230]; ibid Eur. Phys. J. C16 161 (2000) hep-th/0001203].

[109] B. Jurco, S. Schraml, P. Schupp and J. Wess, Eur. Phys. J. C 17521 (2000) [hep-th/0006246].

[110] A. H. Chamseddine, Int. J. Mod. Phys. A 16759 (2001) [hep-th/0010268].

[111] P. Nicolini, J. Phys. A 38 L631 (2005).

[112] S. Ansoldi, P. Nicolini, A. Smailagic, E. Spallucci, Phys. Lett. B 645261 (2007).

[113] S. Fabi, B. Harms, A. Stern Phys. Rev. D 78065037 (2008) arXiv:0808.0943].

[114] V. P. Frolov, M. A. Markov and V. F. Mukhanov Phys. Rev. D 41383 (1990).

[115] I. Dymnikova, Gen. Rel. Grav. 24235 (1992). 
[116] Y. S. Myung, Yong-Wan Kim and Young-Jai Park, JHEP 02012 (2007) [gr-qc/0611130].

[117] R. Banerjee, B. R. Majhi, S. Samanta, Phys. Rev. D 77124035 (2008) arXiv:0801.3583].

[118] C. O. Lousto and N. Sanchez, Phys. Lett. B 212411 (1988).

[119] D. V. Fursaev, Phys. Rev. D 515352 (1995) hep-th/9412161.

[120] J. W. York, Jr., Phys. Rev. D 31755 (1985).

[121] R. Banerjee and B. R. Majhi, Phys. Lett. B 66262 (2008) arXiv:0801.0200. 


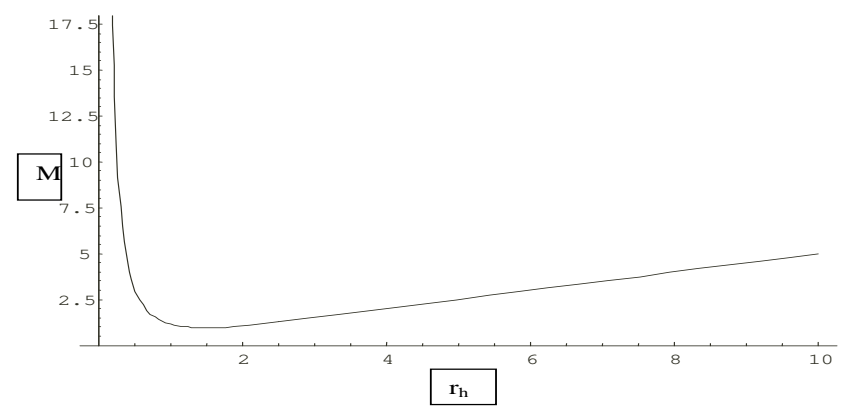

Figure 1: $M V s . r_{h}$ plot.

$M$ is plotted in units of $2 \sqrt{\theta}$ and $r_{h}$ is plotted in units of $2 \sqrt{\theta}$ For eq. (206). 


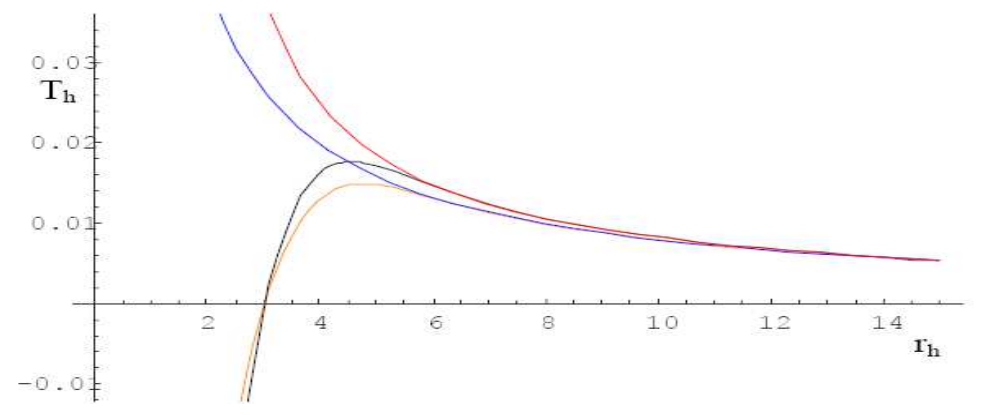

Figure 2: $T_{h}$ Vs. $r_{h}$ plot (Here $\alpha=\theta, \alpha$ and $\theta$ are positive). $r_{h}$ is plotted in units of $\sqrt{\theta}$ and $T_{h}$ is plotted in units of $\frac{1}{\sqrt{\theta}}$. Red curve: $\alpha \neq 0, \theta=0$.

Blue curve: $\alpha=0, \theta=0$.

Black curve: $\alpha \neq 0, \theta \neq 0$.

Yellow curve: $\alpha=0, \theta \neq 0$. 


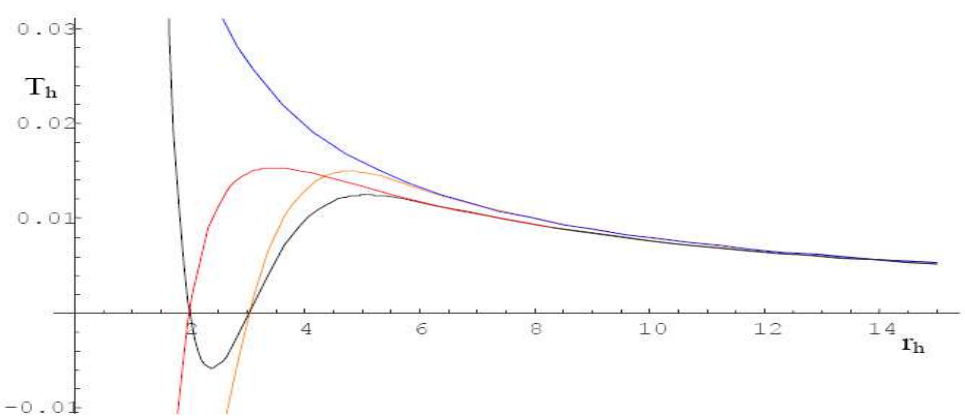

Figure 3: $T_{h}$ Vs. $r_{h}$ plot (Here $|\alpha|=\theta, \alpha$ is negative but $\theta$ is positive). $r_{h}$ is plotted in units of $\sqrt{\theta}$ and $T_{h}$ is plotted in units of $\frac{1}{\sqrt{\theta}}$. Red curve: $\alpha \neq 0, \theta=0$.

Blue curve: $\alpha=0, \theta=0$.

Black curve: $\alpha \neq 0, \theta \neq 0$.

Yellow curve: $\alpha=0, \theta \neq 0$. 


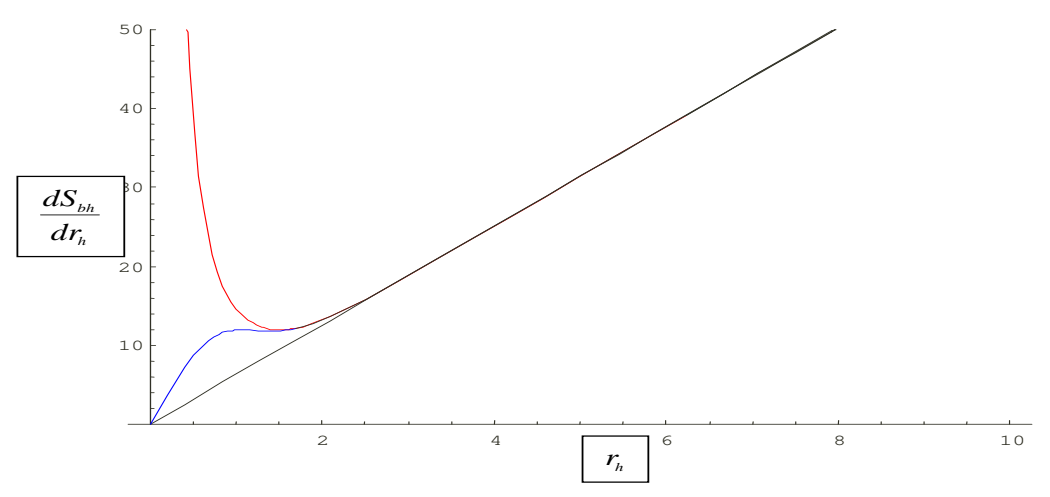

Figure 4: $\frac{d S_{b h}}{d r_{h}}$ Vs. $r_{h}$ plot.

$\frac{d S_{b h}}{d r_{h}}$ is plotted in units of $4 \theta$ and $r_{h}$ is plotted in units of $2 \sqrt{\theta}$

Red curve: for eq. (225)

Black curve: for eq. (226)

Blue curve: for eq. 227) (up to $\Gamma^{2}$ ). 